Supporting Information

\title{
Copper-catalyzed Enantioselective Substitution of Allylic Carbonates with Diboron: An Efficient Route to Optically Active a-Chiral Allylboronates
}

Hajime Ito, * Shinichiro Ito, Yusuke Sasaki, Kou Matsuura, and Masaya Sawamura*

Department of Chemistry, Faculty of Science, Hokkaido University, Sapporo 060-0810, JAPAN

\section{DFT Calculations.}

\section{Computational Methods.}

All calculations were performed with a Gaussian 03 software. ${ }^{1}$ The geometry optimization was performed at the B3LYP level with the $6-31 \mathrm{G}^{* *}$ basis set. Gibbs free energies were obtained on the basis of the calculated frequencies.

\section{Results.}

Calculated potential energies and Gibbs free energies of the relevant intermediates and transition states are shown in Figure S1. Figure S2 shows structures of the selected intermediates and transition states. The energy diagram in Scheme S1 clearly suggests that the catalytic reaction proceeds via the addition-elimination pathway. The first step of the $\mathrm{Cu}(\mathrm{I})$-catalyzed substitution of allylic carbonates substitution is the alkene coordination to the copper center of intermediate B1 forming $\pi$ complex $\mathbf{C 1}$ $(-4.1 \mathrm{kcal} / \mathrm{mol}$ relative to the sum of free energies of $\mathbf{B} \mathbf{1}$ and allyl alcohol). The addition of the B-Cu bond across the $\mathrm{C}-\mathrm{C}$ double bond affords $\beta$-boryl alkylcopper intermediate $\mathbf{D} 1(-13.3 \mathrm{kcal} / \mathrm{mol})$ such that the $\mathrm{Cu}$ and $\mathrm{B}$ atoms are located at the $\beta$ - and $\gamma$-positions, respectively, with low activation energy (+9.2 kcal/mol). An allylboronate and intermediate $\mathbf{A 1}$ are produced through $\beta$-elimination from D1. The overall reaction is highly exergonic by $34.4 \mathrm{kcal} / \mathrm{mol}$. Although attempts to find the transition state of this $\beta$-alkoxy elimination were unsuccessful, it is reasonable to expect that intermediate D1 easily degrades through $\beta$-alkoxy elimination, since it is commonly feasible process of organometallic 
compounds.

Formation of alkylcopper intermediate D2 that does not lead to the allylboronate is kinetically and thermodynamically unfavorable compared to the formation of D1. Although the $\pi$-complex C2 $(-4.7$ $\mathrm{kcal} / \mathrm{mol})$ is slightly stable than $\mathbf{C 1}$, the activation energy of the addition reaction of $\mathbf{C 2}(+14.1$ $\mathrm{kcal} / \mathrm{mol})$ is considerably higher than that of C1. Addition product D2 $(-12.1 \mathrm{kcal} / \mathrm{mol})$ is unstable than D1. Stereoelectronic effects that stabilize the $\sigma(\mathrm{Cu}-\mathrm{C})$ bond through interactions with the $\sigma^{*}(\mathrm{Cu}-\mathrm{O})$ bond contribute to the low energies of TS1 and D1, where $\mathrm{Cu}, \mathrm{C}(\beta), \mathrm{C}(\alpha)$ and $\mathrm{O}$ atoms are in a nearly antiperiplanar alignment (dihedral angles of $\mathrm{Cu}-\mathrm{C}(\beta)-\mathrm{C}(\alpha)-\mathrm{O}$ are $152.2^{\circ}$ and $178.5^{\circ}$, respectively).

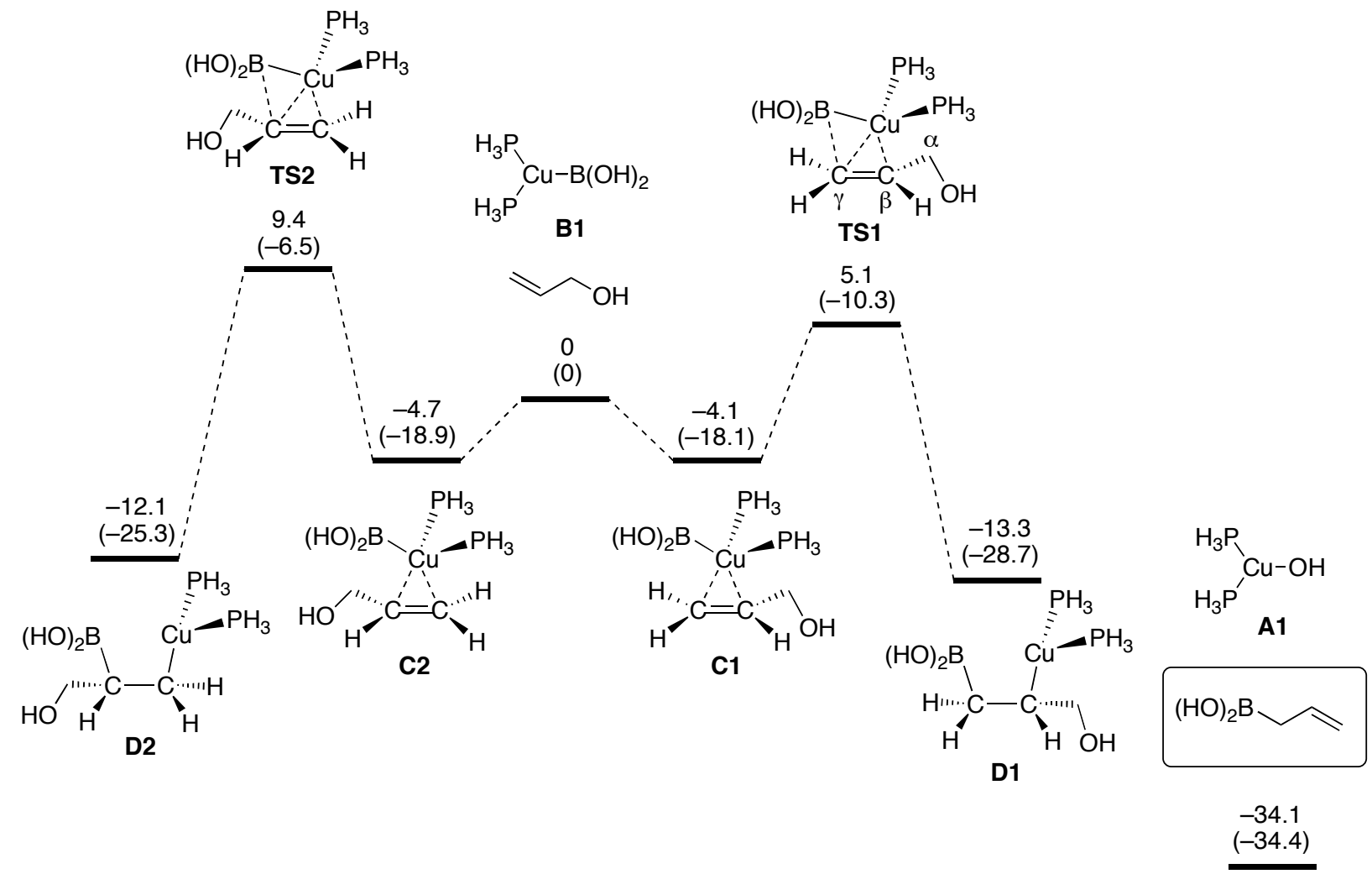

Figure S1. Energy diagram calculated for the $\mathrm{Cu}(\mathrm{I})$-catalyzed substitution of allylic carbonates with diborons. The relative Gibbs free energies and electronic energies (in parentheses) are given in $\mathrm{kcal} / \mathrm{mol}$. 

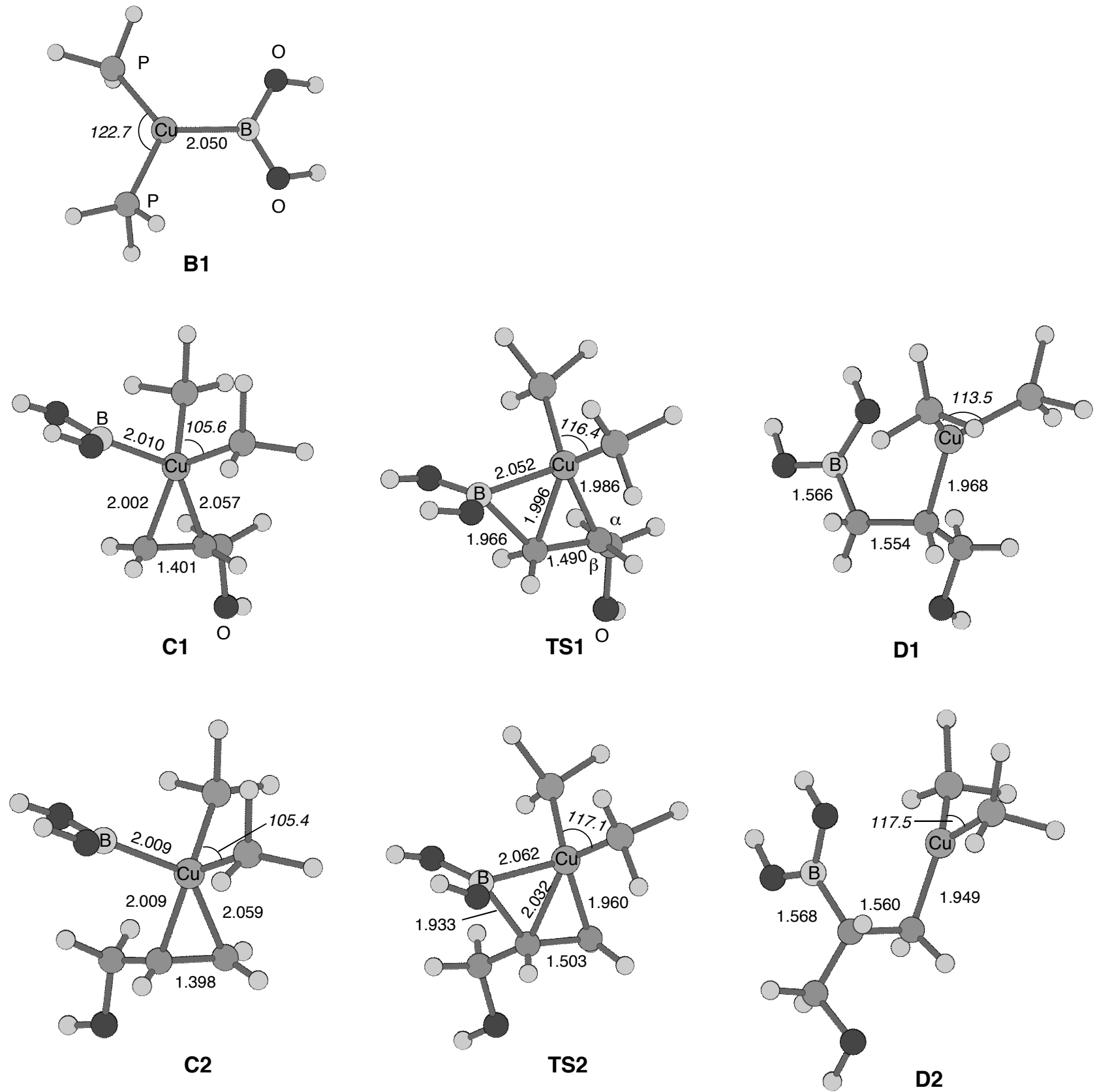

Figure S2. Structures of the selected intermediates and transition states in $\mathrm{Cu}(\mathrm{I})$-catalyzed substitution with diboron. The values refer to bond length $(\AA)$ and bond angles (degrees, italics). 


\section{Experimaental Details}

\section{Caution.}

$\mathrm{CO}_{2}$ gas evolution occurs in the copper(I)-catalyzed allylic substitution. The gas may contain carbon monoxide produced through the reduction of $\mathrm{CO}_{2}$ in the presence of diboron and copper(I) complex. $^{2}$ The experiment should be done in a well-ventilated hood. Adequate care should be taken to avoid injury in large scale experiment.

\section{Materials.}

Materials were obtained from commercial suppliers and purified by the standard procedure unless otherwise noted. $\mathrm{Cu}(\mathrm{O}-t-\mathrm{Bu})$ was prepared from $\mathrm{CuCl}$ and $\mathrm{Na}(\mathrm{O}-t-\mathrm{Bu})$ via Tsuda's procedure. ${ }^{3}$ $\mathrm{Cu}(\mathrm{O}-\mathrm{t}-\mathrm{Bu})$ is highly air sensitive and should be handled in a glove box. Bis(pinacolato)diboron was purchased from AllyChem Co., Ltd. or prepared according to the literarute. ${ }^{4}$ Dry tetrahydrofuran (THF) was purchased from Tokyo Kasei Kogyo Co., Ltd and was degassed via three freeze-pump-thaw cycles and was further dried on MS 4A. THP was purified by distillation over sodium benzophenone ketyl under argon. Toluene and DMI ( $N, N$-dimethyimidazolidinone) were purified by distillation over $\mathrm{CaH}_{2}$ under argon and were stored on MS 4A. $(R, R)$-QuinxP* was purchased from Aldrich Chemical Co. or received from Nippon Chemical Industrial Co., Ltd. Other chiral ligands were purchased from commercial suppliers.

\section{Analysis.}

NMR spectra were recorded on Varian Gemini $2000\left({ }^{1} \mathrm{H}: 300 \mathrm{MHz} ;{ }^{13} \mathrm{C}: 75.4 \mathrm{MHz}\right)$ spectrometer. Tetramethylsilane $\left({ }^{1} \mathrm{H}\right)$ and $\mathrm{CDCl}_{3}\left({ }^{31} \mathrm{C}\right)$ were employed as internal and external standards, respectively. Gas chromatographic (GLC) analyses were conducted on a Shimadzu GC-14B equipped with a flame ionization detector. Infrared spectra were recorded on a Perkin-Elmer Spectrum One. Elemental analyses were performed at the Center for Instrumental Analysis, Hokkaido University. Low- and High-resolution mass spectra were recorded on a JEOL JMS-700TZ mass spectrometer. HPLC analysis was conducted on a Shimadzu LC6A system with a Shimadzu SPD-10A UV detector.

\section{Preparation of allylic alcohols.}

(E)-5-Phenyl-2-peneten-1-ol was prepared according to the literature procedure. ${ }^{5}$ (Z)-5-Phenyl-2-peneten-1-ol, (Z)-2-propen-1-ol, (Z)-2-octen-1-ol and (Z)-4-methyl-2-penten-1-ol were 
prepared from the corresponding propargylic alcohols through the hydrogenation in the presence of Lindlar's catalyst. $^{6-8}$ (Z)-5-Methyl-2-hexen-1-ol was prepared from 5-methyl-2-hexyn-1-ol through the reaction with $\mathrm{Cp}_{2} \mathrm{TiCl}_{2} / i$-BuMgBr${ }^{9}$ (Z)-6-( $t$-Butyldimethylsiloxy)-2-hexen-1-ol was prepared according to the literature procedure. ${ }^{10}$

Allylic alcohols were subjected to purification repeatedly by using column chromatography until the high isomeric purity of the alcohols $(E: Z=1:>20)$ was achieved.

A typical procedure for preparation of allylic carbonates $((Z)-1 \mathrm{a},(E)-1 \mathrm{a},(Z)-1 \mathrm{~b},(Z)-1 \mathrm{c}(Z)-1 \mathrm{~d}$, (Z)-1e, (Z)-1f).

To a solution of allylic alcohol $(4.5 \mathrm{mmol})$ and pyridine $(1.6 \mathrm{~mL}, 20 \mathrm{mmol})$ in dichloromethane $(10$ $\mathrm{mL})$, methyl chloroformate $(0.77 \mathrm{~mL}, 10 \mathrm{mmol})$ was added with stirring at $0^{\circ} \mathrm{C}$. After the reaction was complete, the reaction mixture was added by water and the organic layer was separated. The aqueous layer was extracted twice with hexane. The combined organic layer was washed with brine and dried over sodium sulfate. After removal of the solvents with a rotary evaporator, the residue was purified by column chromatography $\left(\mathrm{SiO}_{2}\right)$. (Z)-allylic carbonates with high stereoisomeric purity $(E: Z=3:>97)$ were obtained by further purification by using flash chromatography $\left(\mathrm{SiO}_{2}\right.$, ethyl acetate:hexane $=0.1: 100-3: 100)$.

\section{(Z)-5-Phenyl-2-penten-1-yl methyl carbonate ((Z)-1a).}

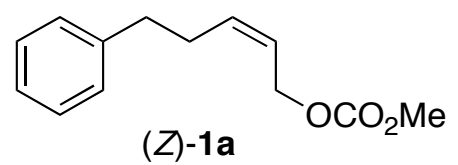

${ }^{1} \mathrm{H}$ NMR (300 MHz, $\left.\mathrm{CDCl}_{3}, \delta\right): 2.44(\mathrm{q}, J=7.4 \mathrm{~Hz}, 2 \mathrm{H}), 2.70(\mathrm{t}, J=7.4 \mathrm{~Hz}, 2 \mathrm{H}), 3.77(\mathrm{~s}, 3 \mathrm{H}), 4.57$ $(\mathrm{d}, J=6.6 \mathrm{~Hz}, 2 \mathrm{H}), 5.52-5.62(\mathrm{~m}, 1 \mathrm{H}), 5.65-5.75(\mathrm{~m}, 1 \mathrm{H}), 7.12-7.33(\mathrm{~m}, 5 \mathrm{H}) . \quad{ }^{13} \mathrm{C} \mathrm{NMR}(75.4 \mathrm{MHz}$, $\left.\mathrm{CDCl}_{3}, \delta\right): 29.3\left(\mathrm{CH}_{2}\right), 35.4\left(\mathrm{CH}_{2}\right), 54.6\left(\mathrm{CH}_{3}\right), 63.4\left(\mathrm{CH}_{2}\right), 123.6(\mathrm{CH}), 126.0(\mathrm{CH}), 128.4(\mathrm{CH}), 128.5$ $(C H), 134.6(C H), 141.3(C), 155.8(C)$. IR (neat, $\mathrm{cm}^{-1}$ ): $3027(w), 1745$ (s), $1253(\mathrm{~s})$. HRMS-ESI $(\mathrm{m} / \mathrm{z})$ : $[\mathrm{M}+\mathrm{Na}]^{+}$calcd for $\mathrm{C}_{13} \mathrm{H}_{16} \mathrm{O}_{3} \mathrm{Na}, 243.09971$; found, 243.09899.

(E)-5-Phenyl-2-penten-1-yl methyl carbonate $((E)-1 a) .{ }^{11}$

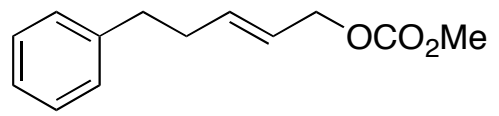

$(E)-1 \mathbf{a}$ 
${ }^{1} \mathrm{H}$ NMR (300 MHz, $\left.\mathrm{CDCl}_{3}, \delta\right): 2.38(\mathrm{q}, J=7.5 \mathrm{~Hz}, 2 \mathrm{H}), 2.71(\mathrm{t}, J=7.5 \mathrm{~Hz}, 2 \mathrm{H}) 3.78(\mathrm{~s}, 3 \mathrm{H}), 4.57$ $(\mathrm{dd}, J=5.5,1.0 \mathrm{~Hz}, 2 \mathrm{H}), 5.57-5.68(\mathrm{~m}, 1 \mathrm{H}), 5.80-5.92(\mathrm{~m}, 1 \mathrm{H}), 7.15-7.32(\mathrm{~m}, 5 \mathrm{H}) . \quad{ }^{13} \mathrm{C}$ NMR $(75.4$ $\left.\mathrm{MHz}, \mathrm{CDCl}_{3}, \delta\right): 33.9\left(\mathrm{CH}_{2}\right), 35.1\left(\mathrm{CH}_{2}\right), 54.7\left(\mathrm{CH}_{3}\right), 68.5\left(\mathrm{CH}_{2}\right), 123.9(\mathrm{CH}), 126.0(\mathrm{CH}), 128.4(\mathrm{CH})$, $128.5(C H), 136.3(C H), 141.5(C), 155.8(C)$. HRMS-ESI $(m / z):[\mathrm{M}+\mathrm{Na}]^{+}$calcd for $\mathrm{C}_{13} \mathrm{H}_{16} \mathrm{O}_{3} \mathrm{Na}$, 243.09971; found, 243.09919.

\section{(Z)-2-Buten-1-yl methyl carbonate $((Z)-1 b)$.}

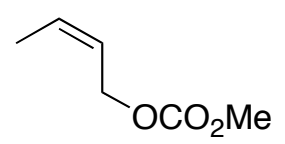

(Z)-1b

${ }^{1} \mathrm{H}$ NMR (300 MHz, $\left.\mathrm{CDCl}_{3}, \delta\right): 1.72(\mathrm{dt}, J=0.8,6.9 \mathrm{~Hz}, 3 \mathrm{H}), 3.79(\mathrm{~s}, 3 \mathrm{H}), 4.71(\mathrm{~d}, J=7.1 \mathrm{~Hz}, 2 \mathrm{H})$, 5.54-5.63 (m, 1H), 5.70-5.80 (m, 1H). $\left.{ }^{13} \mathrm{C} \mathrm{NMR} \mathrm{(75.4} \mathrm{MHz,} \mathrm{CDCl}_{3}, \delta\right): 13.0\left(\mathrm{CH}_{3}\right), 54.6\left(\mathrm{CH}_{3}\right), 63.3$ $\left(\mathrm{CH}_{2}\right), 123.6(\mathrm{CH}), 130.4(\mathrm{CH}), 155.9(\mathrm{C})$. IR (neat, $\left.\mathrm{cm}^{-1}\right): 2959(\mathrm{w}), 1745$ (s), 1251 (s). HRMS-EI $(m / z):[\mathrm{M}+]^{+}$calcd for $\mathrm{C}_{6} \mathrm{H}_{10} \mathrm{O}_{3}, 130.0630$; found, 130.0631 .

(Z)-2-Octen-1-yl methyl carbonate $((Z)-1 \mathrm{c})$.

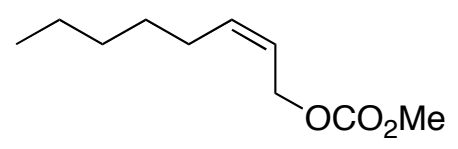

$(Z)-1 c$

${ }^{1} \mathrm{H}$ NMR (300 MHz, $\left.\mathrm{CDCl}_{3}, \delta\right): 0.89(\mathrm{t}, J=6.9 \mathrm{~Hz}, 3 \mathrm{H}), 1.26-1.42(\mathrm{~m}, 6 \mathrm{H}), 2.11(\mathrm{q}, J=6.9 \mathrm{~Hz}$, 2H), $3.79(\mathrm{~s}, 3 \mathrm{H}), 4.69(\mathrm{~d}, J=6.9 \mathrm{~Hz}, 2 \mathrm{H}), 5.51-5.60(\mathrm{~m}, 1 \mathrm{H}), 5.63-5.73(\mathrm{~m}, 1 \mathrm{H}) .{ }^{13} \mathrm{C}$ NMR $(75.4$ $\left.\mathrm{MHz}, \mathrm{CDCl}_{3}, \delta\right): 13.8\left(\mathrm{CH}_{3}\right), 22.3\left(\mathrm{CH}_{2}\right), 27.4\left(\mathrm{CH}_{2}\right), 28.9\left(\mathrm{CH}_{2}\right), 31.2\left(\mathrm{CH}_{2}\right), 54.6\left(\mathrm{CH}_{3}\right), 63.7\left(\mathrm{CH}_{2}\right)$, $122.7(\mathrm{CH}), 136.2(\mathrm{CH}), 155.9(\mathrm{C}) . \mathrm{IR}\left(\right.$ neat, $\left.\mathrm{cm}^{-1}\right): 2929(\mathrm{w}), 1748(\mathrm{~s}), 1253(\mathrm{~s})$. HRMS-EI $(\mathrm{m} / \mathrm{z}):[\mathrm{M}]^{+}$ calcd for $\mathrm{C}_{10} \mathrm{H}_{18} \mathrm{O}_{3}, 186.1256$; found, 186.1254 .

\section{(Z)-5-Methyl-2-hexen-1-yl methyl carbonate ((Z)-1d).}

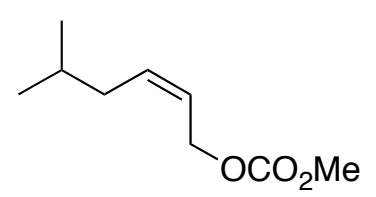

$(Z)-1 d$

${ }^{1} \mathrm{H}$ NMR (300 MHz, $\left.\mathrm{CDCl}_{3}, \delta\right): 0.90(\mathrm{~d}, J=6.6 \mathrm{~Hz}, 6 \mathrm{H}), 1.65(\mathrm{sep}, J=6.8 \mathrm{~Hz}, 1 \mathrm{H}), 2.01(\mathrm{t}, J=6.9$ $\mathrm{Hz}, 2 \mathrm{H}), 3.79(\mathrm{~s}, 3 \mathrm{H}), 4.69(\mathrm{~d}, J=6.3 \mathrm{~Hz}, 2 \mathrm{H}), 5.55-5.74(\mathrm{~m}, 2 \mathrm{H}) .{ }^{13} \mathrm{C} \mathrm{NMR}\left(75.4 \mathrm{MHz}, \mathrm{CDCl}_{3}, \delta\right)$ : 
$22.1\left(\mathrm{CH}_{3}\right), 28.3\left(\mathrm{CH}_{2}\right), 36.4\left(\mathrm{CH}_{2}\right), 54.6\left(\mathrm{CH}_{3}\right), 63.7\left(\mathrm{CH}_{2}\right), 123.5(\mathrm{CH}), 134.8(\mathrm{CH}), 155.9(C)$. IR (neat, $\mathrm{cm}^{-1}$ ): 2957 (w), 1747 (s), 1253 (s). HRMS-EI (m/z): [M+] calcd for $\mathrm{C}_{9} \mathrm{H}_{16} \mathrm{O}_{3}, 172.1099$; found, 172.1100 .

(Z)-4-Methyl-2-penten-1-yl methyl carbonate ((Z)-1e).

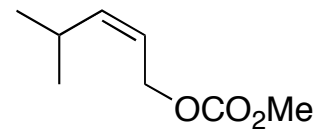

$(Z)-1 e$

${ }^{1} \mathrm{H}$ NMR $\left(300 \mathrm{MHz}, \mathrm{CDCl}_{3}, \delta\right): 0.98(\mathrm{~d}, J=6.6 \mathrm{~Hz}, 6 \mathrm{H}), 2.61-2.70(\mathrm{~m}, 1 \mathrm{H}), 3.79$ (s, 3H), $4.70(\mathrm{~d}$, $J=5.8 \mathrm{~Hz}, 2 \mathrm{H}), 5.38-5.54(\mathrm{~m}, 2 \mathrm{H}) .{ }^{13} \mathrm{C} \mathrm{NMR}\left(75.4 \mathrm{MHz}, \mathrm{CDCl}_{3}, \delta\right): 22.7\left(\mathrm{CH}_{3}\right), 26.8(C \mathrm{H}), 54.5$ $\left(\mathrm{CH}_{3}\right), 63.6\left(\mathrm{CH}_{2}\right), 120.4(\mathrm{CH}), 143.2(\mathrm{CH}), 155.8(C)$. IR (neat, $\left.\mathrm{cm}^{-1}\right)$ : $2960(\mathrm{w}), 1747(\mathrm{~s}), 1253$ (s). HRMS-EI $(m / z):[M+]^{+}$calcd for $\mathrm{C}_{8} \mathrm{H}_{14} \mathrm{O}_{3}, 158.0943$; found, 158.0938 .

(Z)-6-(t-Butyldimethylsiloxy)-2-hexen-1-yl methyl carbonate ((Z)-1f).

TBSO

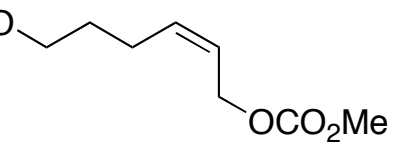

$(Z)-1 f$

${ }^{1} \mathrm{H}$ NMR (300 MHz, $\left.\mathrm{CDCl}_{3}, \delta\right): 0.04$ (s, 6H), 0.89 (s, 9H), 1.59 (quint, $\left.J=7.7 \mathrm{~Hz}, 2 \mathrm{H}\right), 2.19$ (q, $J=$ $7.1 \mathrm{~Hz}, 2 \mathrm{H}), 3.61(\mathrm{t}, J=6.3 \mathrm{~Hz}, 2 \mathrm{H}), 3.78(\mathrm{~s}, 3 \mathrm{H}), 4.70(\mathrm{~d}, J=6.3 \mathrm{~Hz}, 2 \mathrm{H}), 5.50-5.73(\mathrm{~m}, 2 \mathrm{H}) .{ }^{13} \mathrm{C}$ NMR (75.4 MHz, $\left.\mathrm{CDCl}_{3}, \delta\right):-5.5\left(\mathrm{CH}_{3}\right), 18.2(C), 23.7\left(\mathrm{CH}_{2}\right), 25.8\left(\mathrm{CH}_{3}\right), 32.2\left(\mathrm{CH}_{2}\right), 54.6\left(\mathrm{CH}_{3}\right)$, $62.1\left(\mathrm{CH}_{2}\right), 63.6\left(\mathrm{CH}_{2}\right), 123.2(\mathrm{CH}), 135.5(\mathrm{CH}), 155.9(\mathrm{C})$. IR (neat, $\left.\mathrm{cm}^{-1}\right): 2955(\mathrm{w}), 1750(\mathrm{~s}), 1254$ (s). HRMS-EI $(\mathrm{m} / \mathrm{z})$ : $\left[\mathrm{M}-\mathrm{C}_{4} \mathrm{H}_{9}\right]^{+}$calcd for $\mathrm{C}_{10} \mathrm{H}_{19} \mathrm{O}_{4} \mathrm{Si}, 231.1053$; found, 231.1054 .

(Z)-6-Benzoyloxy-2-hexen-1-yl methyl carbonate ((Z)-1g).

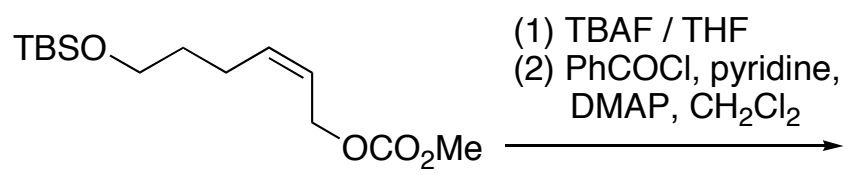

$(Z)-1 f$

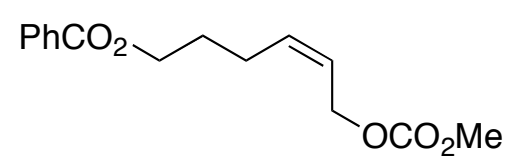

$(Z)-19$

To a solution of (Z)-1f (450 mg, $1.56 \mathrm{mmol})$ in THF (3.0 mL), a THF solution of TBAF (1.0 M, 2.0 $\mathrm{mL}$ ) was added at $0^{\circ} \mathrm{C}$ with stirring. After the reaction was completed, the reaction mixture was added by water and extracted with ethyl acetate three times. The combined organic layer was washed with brine, dried over $\mathrm{Na}_{2} \mathrm{SO}_{4}$, and then evaporated. The residual oil was purified by column 
chromatography $\left(\mathrm{SiO}_{2}\right.$, hexane:ethylacetate $\left.=3: 1\right)$ to give $(Z)$-6-hydroxy-2-hexen-1-yl methyl carbonate (239 mg, $1.37 \mathrm{mmol}, 88 \%)$. ${ }^{1} \mathrm{H}$ NMR (300 MHz, $\mathrm{CDCl}_{3}, \delta$ ): 1.63-1.71 (m, 3H), 1.74-1.83 $(\mathrm{m}, 1 \mathrm{H}), 2.26(\mathrm{q}, J=7.1 \mathrm{~Hz}, 2 \mathrm{H}), 3.62-3.67(\mathrm{~m}, 2 \mathrm{H}), 3.16(\mathrm{~s}, 3 \mathrm{H}), 4.71(\mathrm{~d}, J=6.3 \mathrm{~Hz}, 2 \mathrm{H}), 5.54-5.73$ $(\mathrm{m}, 2 \mathrm{H}) .{ }^{13} \mathrm{C} \mathrm{NMR}\left(75.4 \mathrm{MHz}, \mathrm{CDCl}_{3}, \delta\right): 23.5\left(\mathrm{CH}_{2}\right), 31.8\left(\mathrm{CH}_{2}\right), 54.7\left(\mathrm{CH}_{3}\right), 61.5\left(\mathrm{CH}_{2}\right), 63.5\left(\mathrm{CH}_{2}\right)$, $123.5(\mathrm{CH}), 135.4(\mathrm{CH}), 155.9(C)$.

To a solution of (Z)-6-hydroxy-2-hexen-1-yl methyl carbonate $(220 \mathrm{mg}, 1.26 \mathrm{mmol})$, pyridine $(0.5$ $\mathrm{mL}), N, N$-dimethylaminopyridine $(10 \mathrm{mg})$ in $\mathrm{CH}_{2} \mathrm{Cl}_{2}(1.0 \mathrm{~mL})$, benzoyl chloride (200 $\mathrm{mL}, 1.78$ mmmol) was added at $0^{\circ} \mathrm{C}$ with stirring. After $1 \mathrm{~h}$, the reaction mixture was added by water and extracted with hexane three times. The combined organic layer was washed with brine, dried over $\mathrm{Na}_{2} \mathrm{SO}_{4}$, and then evaporated. The residual oil was purified by column chromatography $\left(\mathrm{SiO}_{2}\right.$, hexane:ethylacetate $=100: 5)$ to give $(Z)-1 g(241 \mathrm{mg}, 0.87 \mathrm{mmol}, 69 \%)$ as a colorless oil.

${ }^{1} \mathrm{H}$ NMR (300 MHz, $\mathrm{CDCl}_{3}, \delta$ ): 1.88 (quint, $\left.J=7.0 \mathrm{~Hz}, 2 \mathrm{H}\right), 2.31$ (q, $\left.J=7.1 \mathrm{~Hz}, 2 \mathrm{H}\right), 3.77$ (s, 3H), $4.34(\mathrm{q}, J=6.5 \mathrm{~Hz}, 2 \mathrm{H}), 4.70(\mathrm{~d}, J=6.3 \mathrm{~Hz}, 2 \mathrm{H}), 5.59-5.77(\mathrm{~m}, 2 \mathrm{H}), 7.26-7.48(\mathrm{~m}, 2 \mathrm{H}), 7.54-7.59(\mathrm{~m}$, 1H), 8.03-8.06 (m, 2H). ${ }^{13} \mathrm{C}$ NMR (75.4 MHz, $\left.\mathrm{CDCl}_{3}, \delta\right): 24.0\left(\mathrm{CH}_{2}\right), 28.2\left(\mathrm{CH}_{2}\right), 54.6\left(\mathrm{CH}_{3}\right), 63.3$ $\left(\mathrm{CH}_{2}\right), 64.0\left(\mathrm{CH}_{2}\right), 123.9(\mathrm{CH}), 128.4(\mathrm{CH}), 129.6(\mathrm{CH}), 130.3(C), 132.9(\mathrm{CH}), 134.5(C \mathrm{H}), 155.8(C)$, $166.6(C)$. IR (neat, $\left.\mathrm{cm}^{-1}\right): 2958(\mathrm{~s}), 1746(\mathrm{~s}), 1716(\mathrm{~s}), 1252$ (s). HRMS-EI $(\mathrm{m} / \mathrm{z}):[\mathrm{M}]^{+}$calcd for $\mathrm{C}_{15} \mathrm{H}_{18} \mathrm{O}_{5}, 278.1154$; found, 278.1155.

(Z)-6-prenyloxy-2-hexen-1-yl methyl carbonate ((Z)-1h).
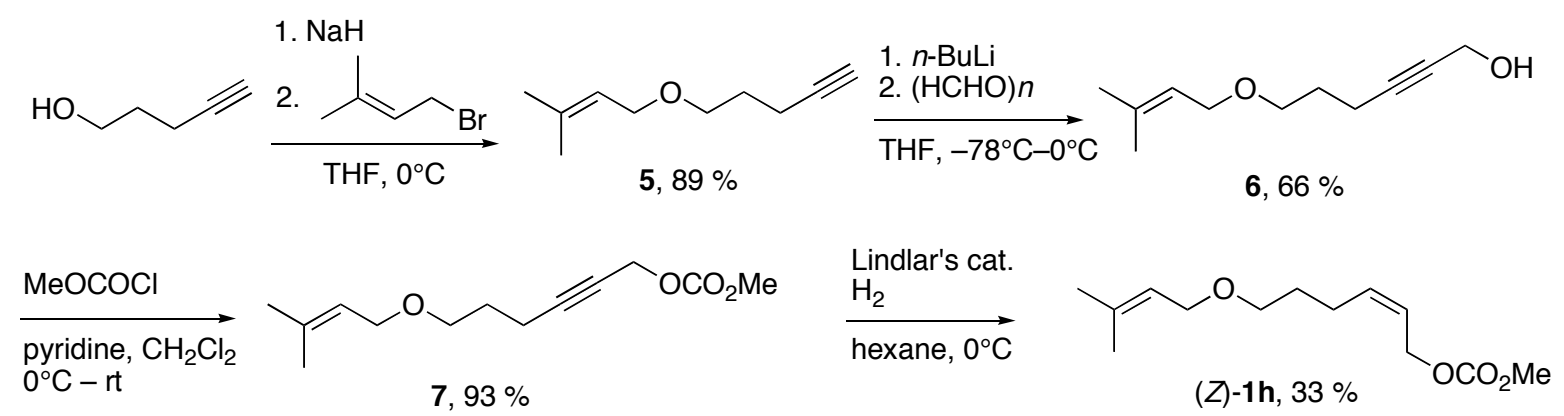

To a suspension of $\mathrm{NaH}(1.32 \mathrm{~g}, 55 \mathrm{mmol})$ in THF (100 mL), 4-pentyn-1-ol (4.62 ml, $4.20 \mathrm{~g}, 50$ $\mathrm{mmol})$ was added dropwise with stirring at $0^{\circ} \mathrm{C}$. After gas evaluation stopped, prenyl bromide $(6.2 \mathrm{~mL}$, $7.87 \mathrm{~g}, 52.5 \mathrm{mmol}$ ) was added dropwise with stirring at $0^{\circ} \mathrm{C}$. The mixture was allowed to warm to room temperature and stirred overnight. After the reaction was quenched with water, the organic layer was separated and the aqueous phase was extracted three times using dichloromethane. The combined organic layer was washed with water and dried over $\mathrm{MgSO}_{4}$, filtered, and evaporated to give 5 (6.76 g, $44 \mathrm{mmol}, 89 \%)$ as a colorless oil. ${ }^{1} \mathrm{H} \mathrm{NMR}\left(300 \mathrm{MHz}, \mathrm{CDCl}_{3}, \delta\right): 1.68$ (s, 3H), $1.75(\mathrm{~s}$, 
$3 \mathrm{H}$ ), 1.80 (quint, $J=6.5 \mathrm{~Hz}, 2 \mathrm{H}), 1.95(\mathrm{t}, J=2.7 \mathrm{~Hz}, 1 \mathrm{H}), 2.30(\mathrm{dt}, J=2.7,7.1 \mathrm{~Hz}, 2 \mathrm{H}), 3.51(\mathrm{t}, J=6.2$ $\mathrm{Hz}, 2 \mathrm{H}), 3.96$ (d, $6.9 \mathrm{~Hz}, 2 \mathrm{H}), 5.32-5.38(\mathrm{~m}, 1 \mathrm{H}) .{ }^{13} \mathrm{C} \mathrm{NMR}\left(75.4 \mathrm{MHz}, \mathrm{CDCl}_{3}, \delta\right): 15.1\left(\mathrm{CH}_{3}\right), 17.8$ $\left(\mathrm{CH}_{3}\right), 25.6\left(\mathrm{CH}_{2}\right), 28.5\left(\mathrm{CH}_{2}\right), 67.2\left(\mathrm{CH}_{2}\right), 68.26(\mathrm{CH}), 68.29\left(\mathrm{CH}_{2}\right), 83.9(C), 121.1(\mathrm{CH}), 136.9(C)$.

$n$-BuLi (1.50 M hexane) was added over $15 \mathrm{~min}$. to a solution of 5 (6.76 g, $44 \mathrm{mmol})$ in THF (50 $\mathrm{mL})$ with stirring at $-78^{\circ} \mathrm{C}$. Then, paraformaldehyde $(1.47 \mathrm{~g}, 46 \mathrm{mmol})$ was added to the reaction mixture in small portions at $-78^{\circ} \mathrm{C}$. The mixture was allowed to warm to room temperature and stirred overnight. After the reaction was quenched with water, the organic layer was separated and the aqueous phase was extracted three times using diethyl ether. The combined organic layer was washed with water and dried over $\mathrm{MgSO}_{4}$, filtered and evaporated. The residue was purified by distillation $\left(1200 \mathrm{~Pa}, 148^{\circ} \mathrm{C}\right)$ to give $6(5.52 \mathrm{~g}, 29.3 \mathrm{mmol}, 66 \%)$ as a colorless oil. ${ }^{1} \mathrm{H}$ NMR $(300 \mathrm{MHz}$, $\left.\mathrm{CDCl}_{3}, \delta\right): 1.68(\mathrm{~s}, 3 \mathrm{H}), 1.75(\mathrm{~s}, 3 \mathrm{H}), 1.78$ (quint, $\left.J=6.6 \mathrm{~Hz}, 2 \mathrm{H}\right), 2.26-2.36(\mathrm{~m}, 2 \mathrm{H}), 3.50(\mathrm{q}, J=6.3$ $\mathrm{Hz}, 2 \mathrm{H}), 3.95(\mathrm{~d}, J=6.9 \mathrm{~Hz}, 2 \mathrm{H}), 4.23-4.27(\mathrm{~m}, 2 \mathrm{H}), 5.30-5.38(\mathrm{~m}, 1 \mathrm{H}) .{ }^{13} \mathrm{C} \mathrm{NMR}\left(75.4 \mathrm{MHz}, \mathrm{CDCl}_{3}\right.$,

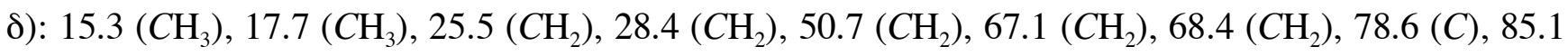
(C), $120.9(\mathrm{CH}), 136.9(C)$.

To a mixture of $6(1.12 \mathrm{~g}, 5.95 \mathrm{mmol})$, pyridine $(1.3 \mathrm{~mL})$ and dichloromethane $(10 \mathrm{~mL})$, methyl chloroformate $(0.62 \mathrm{~mL}, 0.76 \mathrm{~g}, 8.0 \mathrm{mmol})$ was added dropwise with stirring at $0^{\circ} \mathrm{C}$. After stirring for $1 \mathrm{~h}$, the reaction was quenched with water. The organic layer was separated and the aqueous phase was extracted three times using diethyl ether. The combined organic layer was washed with water and dried over $\mathrm{MgSO}_{4}$, filtered and evaporated to give 7 (1.34 g, $\left.5.5 \mathrm{mmol}, 93 \%\right)$ as a colorless oil. ${ }^{1} \mathrm{H}$ NMR (300 MHz, $\mathrm{CDCl}_{3}, \delta$ ): 1.68 (s, 3H), 1.75 (s, 3H), 1.78 (quint, $J=6.3 \mathrm{~Hz}, 2 \mathrm{H}$ ), 2.30-2.36 (m, 2H), $3.48(\mathrm{t}, J=6.3 \mathrm{~Hz}, 2 \mathrm{H}), 3.81(\mathrm{~s}, 3 \mathrm{H}), 3.95(\mathrm{~d}, J=6.9 \mathrm{~Hz}, 2 \mathrm{H}), 4.73(\mathrm{t}, J=2.3 \mathrm{~Hz}, 2 \mathrm{H}), 5.32-5.38$ $(\mathrm{m}, 1 \mathrm{H})$.

The propargylic ester 7 was added to a mixture of Lindlar's catalyst (Aldlich) (0.07 g), quinoline $(0.06 \mathrm{~mL})$ in hexane $(15 \mathrm{~mL})$. The mixture was stirred at $0^{\circ} \mathrm{C}$ under hydrogen atmosphere (hydrogen balloon) for $3 \mathrm{~h}$. The catalyst was filtered off and rinsed with hexane. The filtrate was concentrated, and the residue was subjected to flash chromatography $\left(\mathrm{SiO}_{2}, \mathrm{Et}_{2} \mathrm{O}\right.$ :hexane $\left.=5: 100\right)$. First two fractions were further purified by Kügelrohr distillation to give $(Z)-\mathbf{1 h}(0.352 \mathrm{~g}, 33 \%, Z: E=>97: 3)$. Other fractions contained $(Z)-\mathbf{1 h}(0.70 \mathrm{~g}, 65 \%, Z: E=94: 6)$.

${ }^{1} \mathrm{H}$ NMR (300 MHz, $\left.\mathrm{CDCl}_{3}, \delta\right): 1.60-1.72$ (m, 2H), 1.67 (s, 3H), 1.75 (s, 3H), 2.20 (q, J = 7.2 Hz, $2 \mathrm{H}), 3.40(\mathrm{t}, J=6.5 \mathrm{~Hz}, 2 \mathrm{H}), 3.78(\mathrm{~s}, 3 \mathrm{H}), 3.93(\mathrm{~d}, J=6.9 \mathrm{~Hz}, 2 \mathrm{H}), 4.69$ (d, $J=6.3 \mathrm{~Hz}, 2 \mathrm{H}), 5.30-5.38$ $(\mathrm{m}, 1 \mathrm{H}), 5.53-5.72(\mathrm{~m}, 2 \mathrm{H}) .{ }^{13} \mathrm{C} \mathrm{NMR}\left(75.4 \mathrm{MHz} \mathrm{CDCl}_{3}, \delta\right): 17.8\left(\mathrm{CH}_{3}\right), 24.1\left(\mathrm{CH}_{2}\right), 25.7\left(C \mathrm{H}_{3}\right), 29.2$ $\left(\mathrm{CH}_{2}\right)$, 54.6 $\left(\mathrm{CH}_{3}\right), 63.6\left(\mathrm{CH}_{2}\right), 67.2\left(\mathrm{CH}_{2}\right), 69.1\left(\mathrm{CH}_{2}\right), 121.2(\mathrm{CH}), 123.4(\mathrm{CH}), 135.3(\mathrm{CH}), 136.9(C)$, 
$155.9(C)$. IR (neat, $\left.\mathrm{cm}^{-1}\right)$ : $2933(\mathrm{~s}), 2858(\mathrm{~s}), 1747(\mathrm{~s}), 1256(\mathrm{~s})$. HRMS-EI $(\mathrm{m} / \mathrm{z})$ : [M] $]^{+}$calcd for $\mathrm{C}_{13} \mathrm{H}_{22} \mathrm{O}_{4}, 242.1518$; found, 242.1515 .

\section{Typical procedure for $\mathrm{Cu}(\mathrm{I})$-catalyzed asymmetric reaction $(0.5 \mathrm{mmol}$ scle).}

In a glove box, $\mathrm{Cu}(\mathrm{O}-t-\mathrm{Bu})(3.4 \mathrm{mg}, 0.025 \mathrm{mmol})$ and $(R, R)$-QuinoxP* $(8.4 \mathrm{mg}, 0.025 \mathrm{mmol})$ was placed into a vial and mixed with dry solvent $(0.5 \mathrm{ml})$ with stirring to give a yellow suspension. Bis(pinacolato)diboron 2 (254 $\mathrm{mg}, 1.0 \mathrm{mmol}$ ) was added to the mixture. The color of resultant mixture changed to dark red. After being sealed with a rubber septum, the reaction vial was removed from the glove box and was connected to an argon line through a needle. Allylic carbonate $\mathbf{1}(0.5 \mathrm{mmol})$ was added to the mixture at $0^{\circ} \mathrm{C}$. Gas evolution is not visually observed in the reaction at $0^{\circ} \mathrm{C}$, because the reaction proceed slowly. However, brisk gas evolution is observed when the reaction is carried out at room temperature. (Caution: The gas may contain carbon monoxide). After the mixture was stirred for 20-48 h, the solvent was evaporated under reduced pressure. Then, the residue was passed through a short silica column $(\varnothing 10 \mathrm{~mm} \times 70 \mathrm{~mm}$, ethyl acetate: hexane $=1: 100)$. After the solvent was evaporated, the residual crude product was purified carefully by flash chromatography (Silica Gel $60 \mathrm{~N}$, spherical, neutral, 40-50 $\mu \mathrm{m}$ (KANTO CHEMICAL Co., Inc.), $\varnothing 20 \mathrm{~mm} \times 70 \mathrm{~mm}$, dietyl ether: hexane $=0.5: 100-2: 100)$ to give pure allylboronate 3 . To obtain the allylboronates in a good yield, the chromatography should be completed within $10 \mathrm{~min}$., since the allylboronates are readily hydrolyzed on silica gel. Addition of two or three drops of chloroform in the crude product upon the flash chromatography improves the product separation from the remaining starting material.

\section{$(S)-4,5,5,5$-Tetramethyl-2-[(3S)-5-phenyl-1-penten-3-yl]-1,3,2-dioxaborolane $((S)$-3a).}

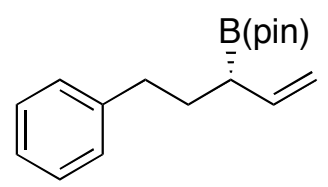

(S)-3a

${ }^{1} \mathrm{H}$ NMR (300 MHz, $\left.\mathrm{CDCl}_{3}, \delta\right): 1.24$ (s, 12H), 1.66-1.83 (m, 1H), 1.83-1.95 (m, 2H), 2.51-2.73 (m, 2H), 4.96-5.07 (m, 2H), 5.75-5.90 (ddd, $J=8.1,10.2,18.6 \mathrm{~Hz}, 1 \mathrm{H}), 7.12-7.30(\mathrm{~m}, 5 \mathrm{H}) .{ }^{13} \mathrm{C}$ NMR (75.4 MHz, $\left.\mathrm{CDCl}_{3}, \delta\right): 24.5\left(\mathrm{CH}_{3}\right), 24.6\left(\mathrm{CH}_{3}\right), 29.8(\mathrm{br}, \mathrm{C}-\mathrm{B}), 32.1\left(\mathrm{CH}_{2}\right), 35.1\left(\mathrm{CH}_{2}\right), 83.2(\mathrm{C}), 114.1$ $\left(\mathrm{CH}_{2}\right), 125.7(\mathrm{CH}), 128.3(\mathrm{CH}), 128.6(\mathrm{CH}), 139.3(\mathrm{CH}), 142.8(\mathrm{C}) .[\alpha]^{29.5}-5.12^{\circ}\left(c 1.04, \mathrm{CHCl}_{3}\right) . \mathrm{IR}$ (neat, $\left.\mathrm{cm}^{-1}\right): 3027(\mathrm{~m}), 2978(\mathrm{~m}), 2928(\mathrm{~m}), 1631(\mathrm{~m}), 1320(\mathrm{~s}), 1141(\mathrm{~s})$. HRMS-EI $(\mathrm{m} / \mathrm{z})$ : $[\mathrm{M}]^{+}$calcd for $\mathrm{C}_{17} \mathrm{H}_{25} \mathrm{O}_{2} \mathrm{~B}, 272.1948$; found, 272.1945 . 
The ee value of this compound was determined by chiral GC analysis of a trifluoroacetate derivative of the alcohol obtained by $\mathrm{H}_{2} \mathrm{O}_{2}$ oxidation of $(S)-3 \mathbf{a}^{11}$ (Chiraldex G-TA, $65^{\circ} \mathrm{C}, S$ isomer $\mathrm{t}_{\mathrm{R}}=$ 79.3 min., $R$ isomer $\mathrm{t}_{\mathrm{R}}=86.2$ min.). The absolute configuration was determined by the Mosher NMR spectroscopic method. Comparison of ${ }^{1} \mathrm{H}$ NMR chemical shifts of Mosher's ester derivatives was illustrated in the following figure.

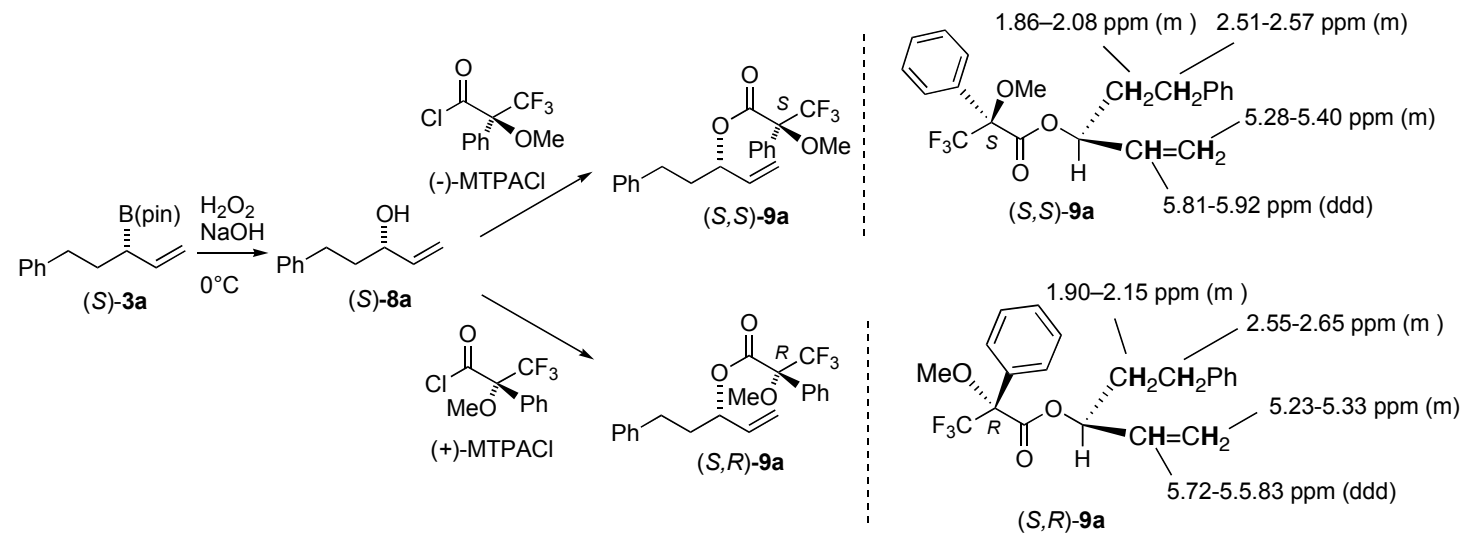

\section{$(S)-4,5,5,5-T e t r a m e t h y l-2-[(3 S)-1-b u t e n-3-y l]-1,3,2-d i o x a b o r o l a n e ~((S)-3 b)$.}

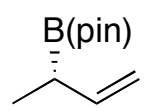

$(S)-3 b$

The reaction of $(Z)-\mathbf{1 b}(520.6 \mathrm{mg}, 4.0 \mathrm{mmol})$ with bis(pinacolato)diboron $2(2.03 \mathrm{~g}, 8.0 \mathrm{mmol})$ was carried out in THF $(4.0 \mathrm{~mL})$ in the presence of $\mathrm{Cu}(\mathrm{O}-t-\mathrm{Bu})(27.3 \mathrm{mg}, 0.2 \mathrm{mmol})$ and $(R, R)-\mathrm{Quinox} *$ $(66.9 \mathrm{mg}, 0.2 \mathrm{mmol})$ at $0^{\circ} \mathrm{C}$ to give $(S)-3 \mathbf{b}(496 \mathrm{mg}, 2.72 \mathrm{mmol}, 68 \%)$ as a colorless oil. ${ }^{1} \mathrm{H}$ NMR (300 MHz, $\left.\mathrm{CDCl}_{3}, \delta\right): 1.10$ (d, $\left.J=7.4 \mathrm{~Hz}, 3 \mathrm{H}\right), 1.24$ (s, 12H), 1.90 (quint, $J=7.3 \mathrm{~Hz}, 1 \mathrm{H}$ ), 4.90-5.01 $(\mathrm{m}, 2 \mathrm{H}), 5.89-6.01(\mathrm{ddd}, J=7.1,10.4,17.3 \mathrm{~Hz}, 1 \mathrm{H}) .{ }^{13} \mathrm{C} \mathrm{NMR}\left(75.4 \mathrm{MHz}, \mathrm{CDCl}_{3}, \delta\right): 13.9\left(C_{3}\right), 22.8$ (br, $\mathrm{CH}-\mathrm{B}), 24.53\left(\mathrm{CH}_{3}\right), 24.56\left(\mathrm{CH}_{3}\right), 83.2(\mathrm{C}), 112.0\left(=\mathrm{CH}_{2}\right), 141.0(-\mathrm{CH}=) .[\alpha]^{14.3}{ }_{\mathrm{D}}-15.6^{\circ}(c) 1.34$, $\mathrm{CHCl}_{3}$ ). IR (neat, $\mathrm{cm}^{-1}$ ): 2979 (m), 1751 (m), 1455 (s), 1323 (s), 1143 (s). HRMS-EI (m/z): [M] calcd for $\mathrm{C}_{10} \mathrm{H}_{19} \mathrm{O}_{2} \mathrm{~B}, 182.1478$; found, 182.1478 .

The ee value was determined by chiral HPLC analysis of the 4-nitrobenzoylated derivative of the corresponding alcohol after $\mathrm{H}_{2} \mathrm{O}_{2} / \mathrm{NaOH}$ oxidation of 3b (Chiralcel AD-H, 0.5\% 2-PrOH/Hexane, 0.5 $\mathrm{mL} / \mathrm{min}$., $R$ isomer $\mathrm{t}_{\mathrm{R}}=26.8$ min., $S$ isomer $\mathrm{t}_{\mathrm{R}}=29.7$ min.). The absolute configuration was determined by HPLC using the 4-nitrobenzoylated derivative of authentic $(S)$-3-buten-1-ol. 
$(S)-4,5,5,5-T e t r a m e t h y l-2-[(3 S)-1-o c t e n-3-y l]-1,3,2-d i o x a b o r o l a n e ~((S)-3 c)$.

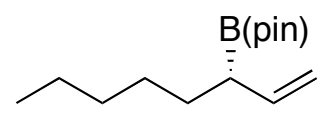

$(S)-3 c$

${ }^{1} \mathrm{H}$ NMR (300 MHz, $\left.\mathrm{CDCl}_{3}, \delta\right): 0.87(\mathrm{t}, J=6.7 \mathrm{~Hz}, 3 \mathrm{H}), 1.24(\mathrm{~s}, 12 \mathrm{H}), 1.20-1.58(\mathrm{~m}, 8 \mathrm{H}), 1.82$ (q, $J=7.9 \mathrm{~Hz}, 1 \mathrm{H}), 4.90-5.10(\mathrm{~m}, 2 \mathrm{H}), 5.71-5.85(\mathrm{ddd}, J=8.8,10.2,19.0 \mathrm{~Hz}, 1 \mathrm{H}) .{ }^{13} \mathrm{C} \mathrm{NMR}(75.4 \mathrm{MHz}$, $\left.\mathrm{CDCl}_{3}, \delta\right): 13.9\left(\mathrm{CH}_{3}\right), 22.4\left(\mathrm{CH}_{2}\right), 24.5\left(\mathrm{CH}_{3}\right), 24.6\left(\mathrm{CH}_{3}\right), 28.5\left(\mathrm{CH}_{2}\right), 30.1\left(\mathrm{CH}_{2}\right) 30.5(\mathrm{br}, C-\mathrm{B}), 31.7$ $\left(\mathrm{CH}_{2}\right) .83 .1(\mathrm{C}-\mathrm{O}), 113.4\left(=\mathrm{CH}_{2}\right), 139.9(-\mathrm{CH}=) .[\alpha]_{\mathrm{D}}^{20}+7.9^{\circ}\left(c\right.$ 1.27, $\left.\mathrm{CHCl}_{3}\right) . \mathrm{IR}$ (neat, $\left.\mathrm{cm}^{-1}\right): 2978(\mathrm{w})$, 2926 (m), $1632(\mathrm{~m}), 1358(\mathrm{~m}), 1320(\mathrm{~m}), 1143(\mathrm{~m})$. HRMS-EI $(\mathrm{m} / \mathrm{z})$ : $[\mathrm{M}]^{+}$calcd for $\mathrm{C}_{14} \mathrm{H}_{27} \mathrm{O}_{2} \mathrm{~B}$, 238.2104; found, 238.2099.

The absolute configuration of this compound was determined by optical rotatory power of the corresponding alcohol $\left([\alpha]_{D}^{20}+9.1^{\circ}\left(c 0.94, \mathrm{CHCl}_{3}\right),\left(\mathrm{lit}^{12,13} \cdot[\alpha]_{\mathrm{D}}^{19}+10.1^{\mathrm{o}}\left(c 0.67, \mathrm{CHCl}_{3}\right),+11.8^{\circ}(c\right.\right.$ $\left.1.02, \mathrm{CHCl}_{3}\right)$ ) obtained by $\mathrm{H}_{2} \mathrm{O}_{2} / \mathrm{NaOH}$ oxidation of $(S)-3 \mathbf{c}$. The ee value was determined by chiral GC analysis of trifluoroacetylated derivative of the alcohol (Chiraldex G-TA, $45^{\circ} \mathrm{C}, S$ isomer $\mathrm{t}_{\mathrm{R}}=12.2 \mathrm{~min}$., $R$ isomer $\mathrm{t}_{\mathrm{R}}=14.6 \mathrm{~min}$.).

$(S)-4,5,5,5-T e t r a m e t h y l-2-[(3 S)-5-m e t h y l-1-h e x e n-3-y l]-1,3,2-d i o x a b o r o l a n e ~((S)-3 d)$.

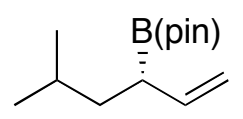

$(S)-3 d$

${ }^{1} \mathrm{H}$ NMR (300 MHz, $\left.\mathrm{CDCl}_{3}, \delta\right): 0.85(\mathrm{~d}, J=6.6 \mathrm{~Hz}, 3 \mathrm{H}), 0.88(\mathrm{~d}, J=6.6 \mathrm{~Hz}, 3 \mathrm{H}), 1.23(\mathrm{~s}, 12 \mathrm{H})$, 1.33-1.42 (m, 2H), $1.59(\mathrm{sep}, J=6.6 \mathrm{~Hz}, 1 \mathrm{H}), 1.94(\mathrm{q}, J=8.2 \mathrm{~Hz}, 1 \mathrm{H}), 4.89-5.05$ (m, 2H), 5.67-5.78 $(\mathrm{m}, 1 \mathrm{H}) .{ }^{13} \mathrm{C}$ NMR $\left(75.4 \mathrm{MHz}, \mathrm{CDCl}_{3}, \delta\right): 22.3\left(\mathrm{CH}_{3}\right), 24.96\left(\mathrm{CH}_{3}\right), 25.02\left(\mathrm{CH}_{3}\right), 27.2(\mathrm{CH}), 39.6$ $\left(\mathrm{CH}_{2}\right), 83.6(\mathrm{C}), 113.9(\mathrm{CH}), 140.3(\mathrm{CH}) . \mathrm{IR}$ (neat, $\left.\mathrm{cm}^{-1}\right): 2979(\mathrm{~m}), 1632(\mathrm{~m}), 1320(\mathrm{~s}), 1142(\mathrm{~s})$. $[\alpha]_{D}^{26.0}+18.8^{\circ}\left(c\right.$ 1.06, $\left.\mathrm{CHCl}_{3}\right)$. HRMS-EI $(\mathrm{m} / \mathrm{z})$ : $[\mathrm{M}]^{+}$calcd for $\mathrm{C}_{13} \mathrm{H}_{25} \mathrm{O}_{2} \mathrm{~B}, 224.1948$; found, 224.1939.

The ee value was determined by chiral HPLC analysis of the 4-nitrobenzoylated derivative of the corresponding alcohol after $\mathrm{H}_{2} \mathrm{O}_{2} / \mathrm{NaOH}$ oxidation of 3d (Chiralcel AD-H, 0.1\% 2-PrOH/Hexane, 0.5 $\mathrm{mL} / \mathrm{min}$., $R$ isomer $\mathrm{t}_{\mathrm{R}}=49.3 \mathrm{~min}$., $S$ isomer $\mathrm{t}_{\mathrm{R}}=52.4 \mathrm{~min}$.). The absolute configuration was determined by the Mosher NMR spectroscopic method. Comparison of ${ }^{1} \mathrm{H}$ NMR chemical shifts of Mosher's ester derivatives was illustrated in the following figure. 


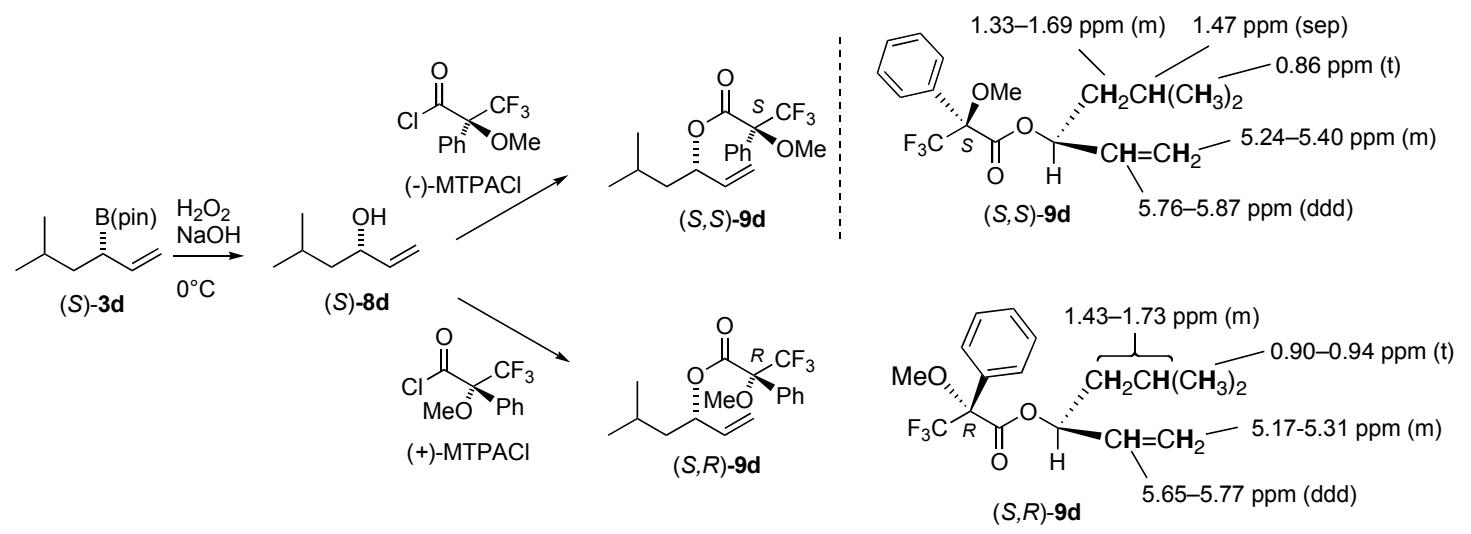

(S)-4,5,5,5-Tetramethyl-2-[(3S)-6-(t-butyldimethylsiloxy)-1-hexen-3-yl]-1,3,2-dioxaborolane ((S)-3f).

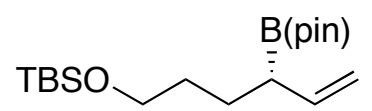

$(S)-3 f$

${ }^{1} \mathrm{H} \mathrm{NMR}\left(300 \mathrm{MHz}, \mathrm{CDCl}_{3}, \delta\right): 0.40$ (s, 6H), 0.89 (s, 9H), 1.24 (s, 12H), 1.38-1.59 (m, 4H), 1.76-1.87 (m, 1H), 3.55-3.65 (m, 2H), 4.91-5.02 (m, 2H), 5.71-5.85 (ddd, $J=8.5,10.2,18.7 \mathrm{~Hz}, 1 \mathrm{H})$. ${ }^{13} \mathrm{C}$ NMR (75.4 MHz, $\left.\mathrm{CDCl}_{3}, \delta\right):-5.4\left(\mathrm{CH}_{3}\right), 18.2(C), 24.5\left(\mathrm{CH}_{3}\right), 24.6\left(\mathrm{CH}_{3}\right), 25.9\left(\mathrm{CH}_{3}\right), 26.2\left(\mathrm{CH}_{2}\right)$, 29.8 (br, $C \mathrm{H}-\mathrm{B}), 32.1\left(\mathrm{CH}_{2}\right), 63.2\left(\mathrm{CH}_{2}\right), 83.1(\mathrm{C}), 113.7(\mathrm{CH}), 139.6(\mathrm{CH}) . \mathrm{IR}$ (neat, $\left.\mathrm{cm}^{-1}\right): 2930(\mathrm{~m})$, 1753(m), $1632(\mathrm{~m}), 1097(\mathrm{~s}) .[\alpha]_{\mathrm{D}}^{25.2}+5.0^{\circ}\left(\mathrm{c} 0.98, \mathrm{CHCl}_{3}\right) . \mathrm{HRMS}-\mathrm{EI}(\mathrm{m} / \mathrm{z}):\left[\mathrm{M}-\mathrm{CH}_{3}\right]^{+}$calcd for $\mathrm{C}_{17} \mathrm{H}_{34} \mathrm{O}_{3} \mathrm{SiB}, 325.2370$; found, 325.2379.

The ee value was determined by chiral HPLC analysis (Chiralcel OD-H, 0.1\% 2-PrOH/Hexane, 0.5 $\mathrm{mL} / \mathrm{min}$., $R$ isomer $\mathrm{t}_{\mathrm{R}}=29.8 \mathrm{~min} ., S$ isomer $\mathrm{t}_{\mathrm{R}}=32.1 \mathrm{~min}$.) of the 4-nitrobenzoylated derivative of the corresponding alcohol after $\mathrm{H}_{2} \mathrm{O}_{2} / \mathrm{NaOH}$ oxidation of $(S)$-3f. The absolute configuration was determined by the Mosher NMR spectroscopic method. Comparison of ${ }^{1} \mathrm{H}$ NMR chemical shifts of Mosher's ester derivatives was illustrated in the following figure. 


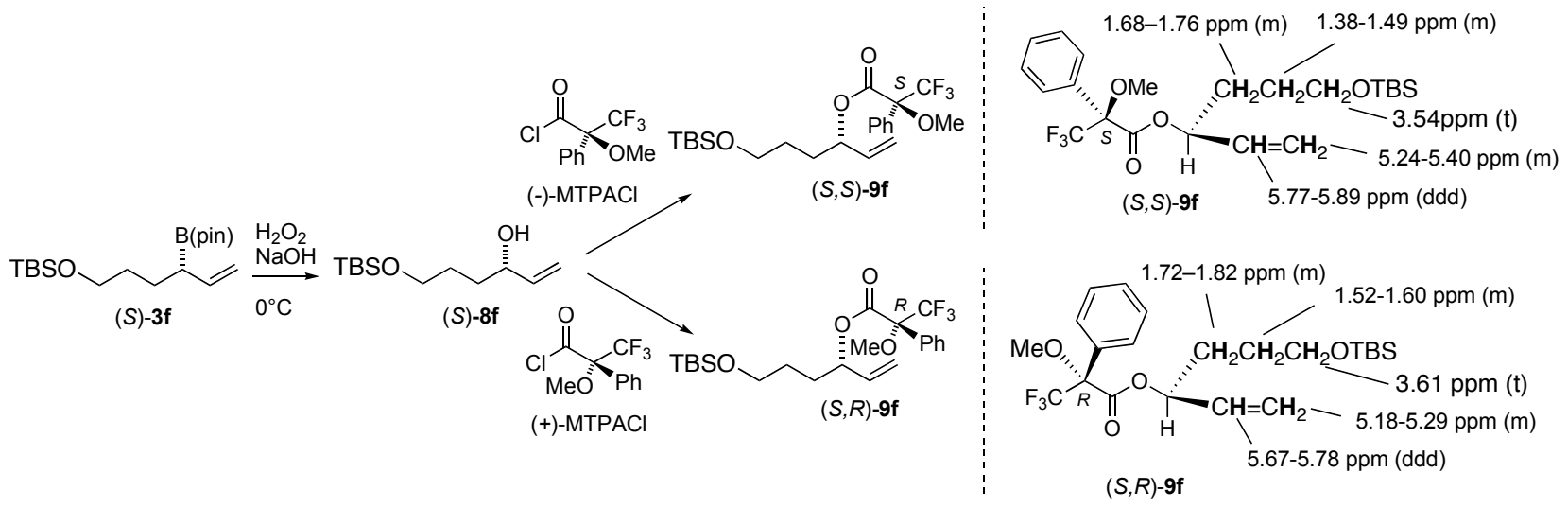

$(S)-4,5,5,5-T e t r a m e t h y l-2-[(3 S)-6-b e n z o y l o x y-1-h e x e n-3-y l]-1,3,2-d i o x a b o r o l a n e ~((S)-3 g)$.<smiles>C=CC(Br)CCCCOC(=O)c1ccccc1</smiles>

(S)-3g

${ }^{1} \mathrm{H}$ NMR (300 MHz, $\left.\mathrm{CDCl}_{3}, \delta\right): 1.24(\mathrm{~s}, 12 \mathrm{H}), 1.55-1.90(\mathrm{~m}, 5 \mathrm{H}), 4.31(\mathrm{t}, J=6.3 \mathrm{~Hz}, 2 \mathrm{H})$, 4.95-5.10 (m, 2H), 5.73-5.87 (ddd, $J=8.5,10.1,18.7 \mathrm{~Hz}, 1 \mathrm{H}), 7.40-7.46(\mathrm{~m}, 2 \mathrm{H}), 7.52-7.59(\mathrm{~m}, 1 \mathrm{H})$, 8.03-8.07 (m, 2H). ${ }^{13} \mathrm{C}$ NMR (75.4 MHz, $\left.\mathrm{CDCl}_{3}, \delta\right): 24.5\left(\mathrm{CH}_{3}\right), 24.6\left(\mathrm{CH}_{3}\right), 26.4\left(\mathrm{CH}_{2}\right), 28.0\left(\mathrm{CH}_{2}\right)$, 29.8 (brs, $C H-B), 65.0\left(C_{2}\right), 83.3(C), 114.1\left(C_{2}\right), 128.3(C H), 129.6(C H), 130.6(C), 132.8(C H)$, $139.1(C H), 166.8(C)$. IR (neat, $\left.\mathrm{cm}^{-1}\right): 2977(\mathrm{~m}), 1718(\mathrm{~s}), 1632(\mathrm{w}), 1602(\mathrm{w}), 1270(\mathrm{~s}) .[\alpha]_{\mathrm{D}}^{26.5}+4.3^{\circ}$ (c 1.15, $\mathrm{CHCl}_{3}$ ). HRMS-EI $(\mathrm{m} / \mathrm{z})$ : $[\mathrm{M}]^{+}$calcd for $\mathrm{C}_{19} \mathrm{H}_{27} \mathrm{O}_{4} \mathrm{~B}, 330.2002$; found, 330.2011 .

The ee value was determined by chiral HPLC analysis (Chiralcel OD-H, 2\% 2-PrOH/Hexane, 0.5 $\mathrm{mL} / \mathrm{min} ., \quad R$ isomer $\mathrm{t}_{\mathrm{R}}=29.9$ min., $S$ isomer $\mathrm{t}_{\mathrm{R}}=32.0 \mathrm{~min}$.) of the corresponding alcohol after $\mathrm{H}_{2} \mathrm{O}_{2} / \mathrm{NaOH}$ oxidation of $(S)$-3g. The absolute configuration was determined by the Mosher NMR spectroscopic method. Comparison of ${ }^{1} \mathrm{H}$ NMR chemical shifts of Mosher's ester derivatives was illustrated in the following figure.
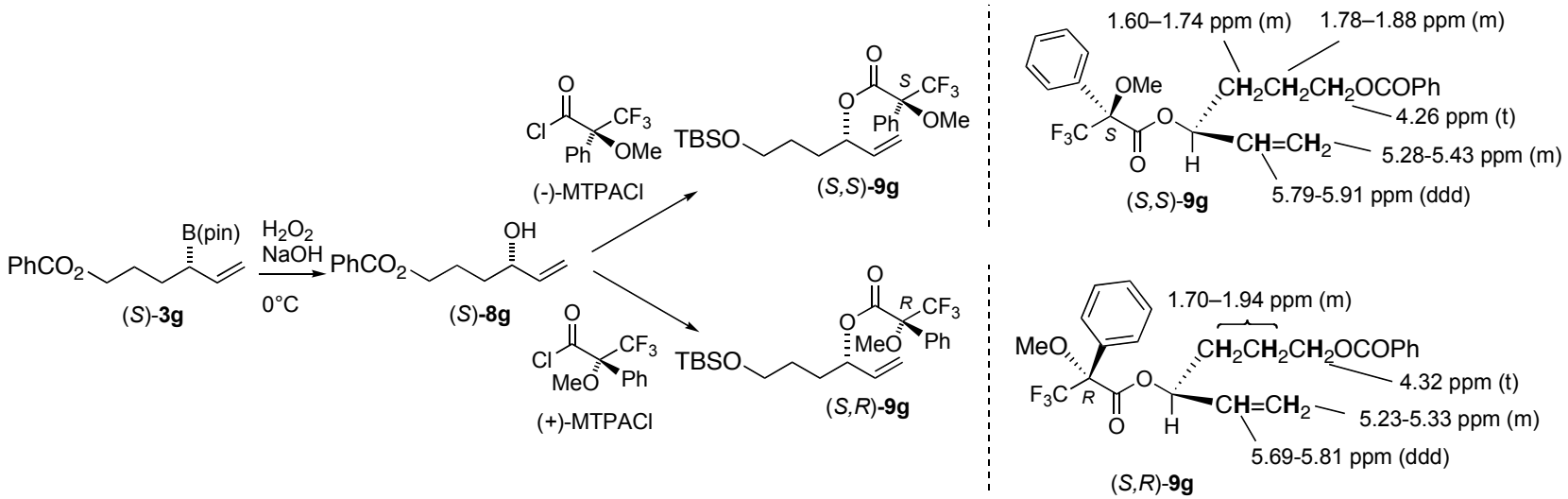
$(S)-4,5,5,5$-Tetramethyl-2-[(3S)-6-prenyloxy-1-hexen-3-yl]-1,3,2-dioxaborolane ((S)-3h).<smiles>C=CC(Br)(Br)CCCCOCC=C(C)C</smiles>

(S) $-3 \mathrm{~h}$

${ }^{1} \mathrm{H}$ NMR (300 MHz, $\left.\mathrm{CDCl}_{3}, \delta\right): 1.23$ (s, 12H), 1.45-1.67 (m, 4H), 1.67 (s, 3H), 1.74 (s, 3H), 1.75-1.88 (m, 1H), 3.35-3.45 (m, 2H), $3.93(\mathrm{~d}, J=6.9 \mathrm{~Hz}, 2 \mathrm{H}), 4.92-5.02(\mathrm{~m}, 2 \mathrm{H}), 5.31-5.39(\mathrm{~m}, 1 \mathrm{H})$, 5.71-5.84 (ddd, $J=8.8,10.2,19.0 \mathrm{~Hz}, 1 \mathrm{H}) .{ }^{13} \mathrm{C} \mathrm{NMR}\left(75.4 \mathrm{MHz}, \mathrm{CDCl}_{3}, \delta\right): 17.9\left(\mathrm{CH}_{3}\right), 24.5\left(\mathrm{CH}_{3}\right)$, $24.6\left(\mathrm{CH}_{3}\right), 25.7\left(\mathrm{CH}_{3}\right), 26.5\left(\mathrm{CH}_{2}\right), 29.0\left(\mathrm{CH}_{2}\right), 30.0$ (brs), $67.1\left(\mathrm{CH}_{2}\right), 70.2\left(\mathrm{CH}_{2}\right), 83.1(C), 113.8$, $121.4(\mathrm{CH}), 136.6(C), 139.4(\mathrm{CH})$. IR (neat, $\left.\mathrm{cm}^{-1}\right)$ : $2977(\mathrm{~m}), 2859(\mathrm{~m}), 1632(\mathrm{~m}), 1321(\mathrm{~s}), 1143(\mathrm{~s})$. $[\alpha]_{\mathrm{D}}^{24.7}+4.7^{\circ}\left(\mathrm{c}\right.$ 1.06, $\left.\mathrm{CHCl}_{3}\right)$; HRMS-EI $(\mathrm{m} / \mathrm{z})$ : $[\mathrm{M}]^{+}$calcd for $\mathrm{C}_{17} \mathrm{H}_{31} \mathrm{O}_{3} \mathrm{~B}, 294.2366$; found, 294.2361.

The ee value was determined by chiral HPLC analysis (Chiralcel OD-H, 0.5\% 2-PrOH/Hexane, 0.5 $\mathrm{mL} / \mathrm{min}$., $R$ isomer $\mathrm{t}_{\mathrm{R}}=35.0$ min., $S$ isomer $\mathrm{t}_{\mathrm{R}}=39.4 \mathrm{~min}$.) of the 4-nitrobenzoylated derivative of the corresponding alcohol after $\mathrm{H}_{2} \mathrm{O}_{2} / \mathrm{NaOH}$ oxidation of $(S)$-3h. The absolute configuration was determined by the Mosher NMR spectroscopic method. Comparison of ${ }^{1} \mathrm{H}$ NMR chemical shifts of Mosher's ester derivatives was illustrated in the following figure.

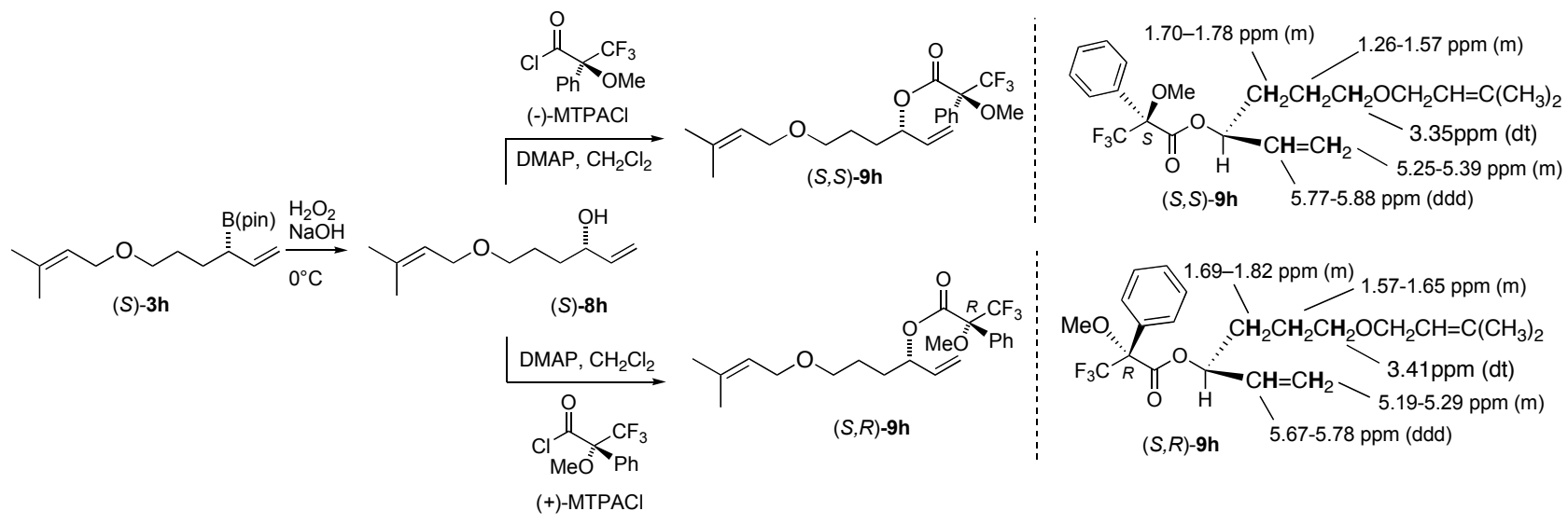

Lewis acid-promoted reaction of allylboronate $(S)-3 c$ with benzaldehyde.

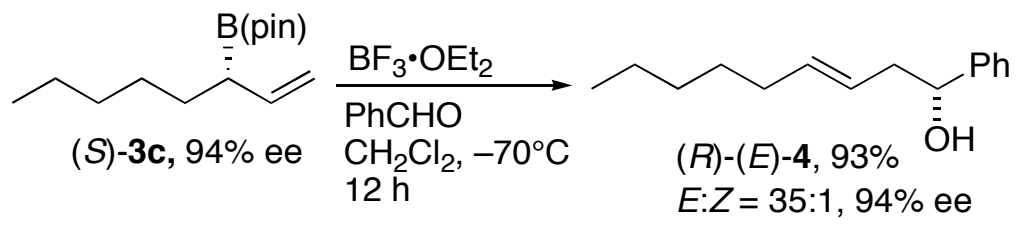

At $-70^{\circ} \mathrm{C}$, to a stirred solution of $(S)-3 \mathbf{c}(49 \mathrm{mg}, 0.206 \mathrm{mmol}, 94 \%$ ee $)$ in $\mathrm{CH}_{2} \mathrm{Cl}_{2}(1 \mathrm{~mL})$, was added 
benzaldehyde $(22 \mu \mathrm{L}, 0.216 \mathrm{mmol})$ and $\mathrm{BF}_{3}-\mathrm{Et}_{2} \mathrm{O}(28 \mu \mathrm{L}, 0.223 \mathrm{mmol})$. After the reaction mixture was further stirred for $12 \mathrm{~h}$ at $-70^{\circ} \mathrm{C}$, aqueous $\mathrm{NaHCO}_{3}$ was added and then allowed to warm to $\mathrm{rt}$. The reaction mixture was extracted with ether, dried over $\mathrm{Na}_{2} \mathrm{SO}_{4}$, and filtered. After the removal of the solvents, the residue was purified by column chromatography $\left(\mathrm{SiO}_{2}\right.$, hexane: ethyl acetate $\left.=95: 5\right)$ to give $(R)-(E)-4(42 \mathrm{mg}, 0.192 \mathrm{mmol}, 93 \%)$ as a colorless oil. The NMR spectra of this compound were identical with those reported. ${ }^{14,15}{ }^{1} \mathrm{H} \mathrm{NMR}\left(300 \mathrm{MHz}, \mathrm{CDCl}_{3}, \delta\right): 0.88$ (t, $\left.J=7.1 \mathrm{~Hz}, 3 \mathrm{H}\right)$, $1.20-1.40$ (m, 6H), 2.01 (q, $J=6.9 \mathrm{~Hz}, 2 \mathrm{H}), 2.08$ (brs, 1H), 2.33-2.51 (m, 2H), 4.64-4.71 (m, 1H), 5.34-5.44 (m, 1H), 5.53-5.64 (m, 1H), 7.23-7.36 (m, 5H). ${ }^{13} \mathrm{C}$ NMR (75.4 MHz, CDCl 3 , $): 13.9\left(C_{3}\right)$, $22.4\left(\mathrm{CH}_{2}\right), 28.9\left(\mathrm{CH}_{2}\right), 31.2\left(\mathrm{CH}_{2}\right), 32.5\left(\mathrm{CH}_{2}\right), 42.7\left(\mathrm{CH}_{2}\right), 73.4(\mathrm{CH}), 125.4(\mathrm{CH}), 125.9(\mathrm{CH}), 127.4$ (CH), $128.4(C H), 135.4(C), 144.1(C H) . ~ I R ~\left(n e a t, \mathrm{~cm}^{-1}\right): 3364($ br), $3064(\mathrm{~m}), 2925(\mathrm{~m}), 1604(\mathrm{~m})$, $1454(\mathrm{~m}) .[\alpha]_{D}^{25}+50.0^{\circ}\left(c=1.03, \mathrm{CHCl}_{3}\right)$. HRMS-EI $(\mathrm{m} / \mathrm{z})$ : calcd for $\mathrm{C}_{15} \mathrm{H}_{22} \mathrm{O}$ : 218.1671; found: 218.1682 .

Enantiomeric purity (94\% ee) and $E / Z$ ratio $(35: 1)$ of the product were determined by HPLC analysis (Chiralcel OD-H, 3\% 2-PrOH/Hexane, $0.5 \mathrm{~mL} / \mathrm{min}$., $R$ isomer $\mathrm{t}_{\mathrm{R}}=16.3 \mathrm{~min}$., $S$ isomer $\mathrm{t}_{\mathrm{R}}=$ 19.4 min., $Z$ isomers, $t_{\mathrm{R}}=17.9$ and 20.9 min.). The absolute configuration was determined by the Mosher NMR spectroscopic method. Comparison of ${ }^{1} \mathrm{H}$ NMR chemical shifts of Mosher's ester derivatives was illustrated in the following figure.
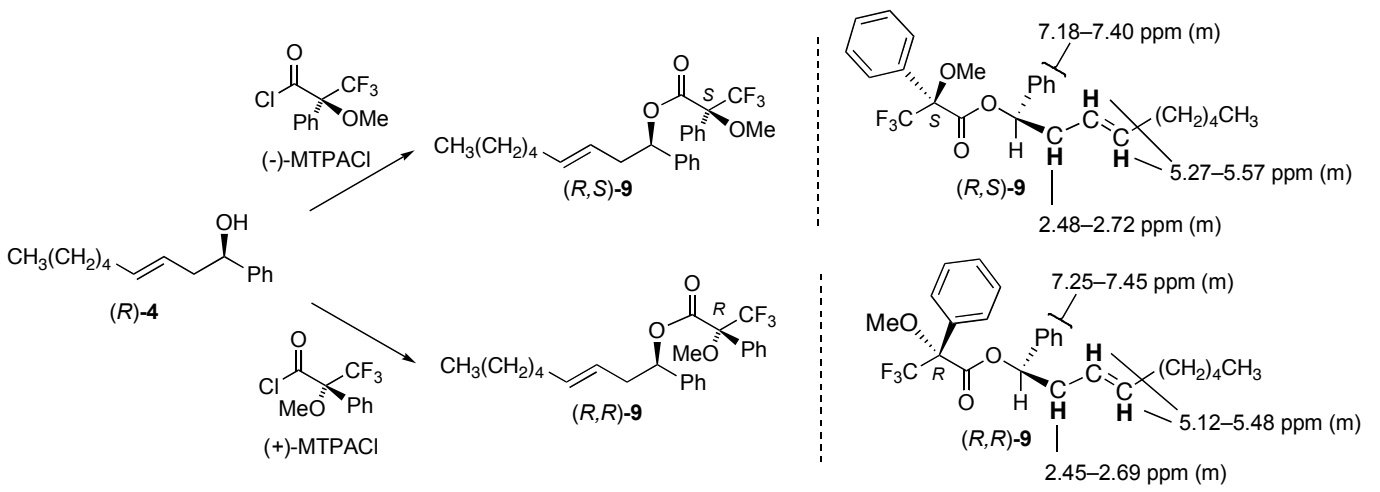


\section{References}

(1) Gaussian 03, Revision C. 02; Frisch, M. J. et al.; Gaussian, Inc.: Pittsburgh, PA, 2003.

(2) Laitar, D. S.; Muller, P.; Sadighi, J. P. J. Am. Chem. Soc. 2005, 127, 17196-17197.

(3) Tsuda, T.; Hashimoto, T.; Saegusa, T. J. Am. Chem. Soc. 1972, 94, 658-659.

(4) Ishiyama, T.; Murata, M.; Ahiko, T.; Miyaura, N. Org. Synth. 1999, 77, 176-181.

(5) Martyn, D. C.; Hoult, D. A.; Abell, A. D. Aust. J. Chem. 2001, 54, 391-396.

(6) Khorana, N.; Purohit, A.; Herrick-Davis, K.; Teitler, M.; Glennon, R. A. Bioorg. Med. Chem. 2003, 11, 717-722.

(7) Fristrup, P.; Jensen, T.; Hoppe, J.; Norrby, P. O. Chem.-Eur. J. 2006, 12, 5352-5360.

(8) Schomaker, J. M.; Pulgam, V. R.; Borhan, B. J. Am. Chem. Soc. 2004, 126, 13600-13601.

(9) Sato, F.; Ishikawa, H.; Sato, M. Tetrahedron Lett. 1981, 22, 85-88.

(10) Garcia-Fandino, R.; Aldegunde, M. J.; Codesido, E. M.; Castedo, L.; Granja, J. R. J. Org. Chem. 2005, 70, 8281-8290.

(11) Ito, H.; Kawakami, C.; Sawamura, M. J. Am. Chem. Soc. 2005, 127, 16034-16035.

(12) Kusuda, S.; Ueno, Y.; Toru, T. Tetrahedron 1994, 50, 1045-1062.

(13) Kang, S. K.; Kim, S. G.; Cho, D. G.; Jeon, J. H. Synth. Commun. 1993, 23, 681-684.

(14) Katritzky, A. R.; Wu, H.; Xie, L. G. J. Org. Chem. 1996, 61, 4035-4039.

(15) Nokami, J.; Nomiyama, K.; Matsuda, S.; Imai, N.; Kataoka, K. Angew. Chem. Int. Ed. 2003, $42,1273-1276$. 


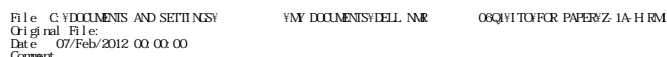

Date
Coment
STANDARD $1 H$ OBSERVE

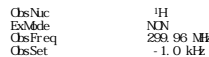

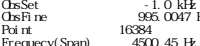

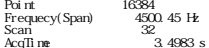

$\begin{array}{ll}\text { AcqTi ne } & 3.4983 \\ \text { PD } & 1.502 \\ \text { Pul se1 } & 6.02\end{array}$

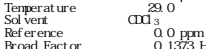

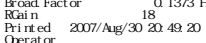
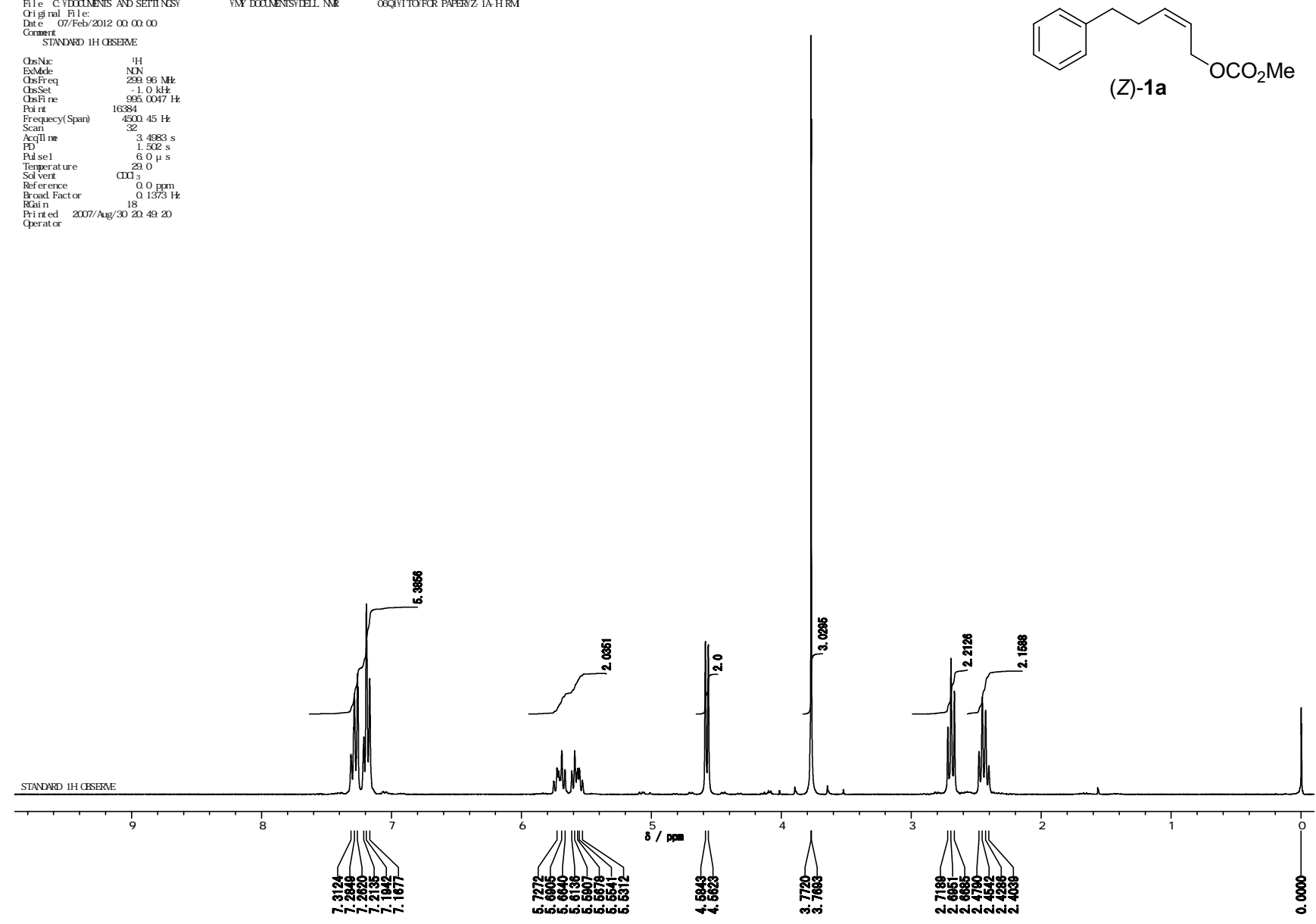

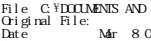

Comment
C13 St at dard dosserve
Sti cknnone Tune-6. 4 Nat che $=0.4$

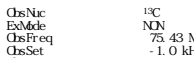

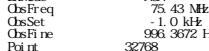

$\begin{array}{ll}\text { Poi ht } & 32768 \\ \text { Frequecy (Span) } & 18761.73 \mathrm{~Hz} \\ \text { Scan } & 256.7 \mathrm{H}\end{array}$

$\begin{array}{lr}\text { Scan } & 256 \\ \text { Acatine } & 1.4992 \mathrm{~s} \\ \text { PD } & 1.501 \mathrm{~s} \\ \text { Pul se1 } & 6.01 \mathrm{~s} \\ \text { Pul } & \end{array}$

Tul ser
Solver ture
Solvt

Sol vent
Ref erence
Broad. Fact or

(Z)-1a
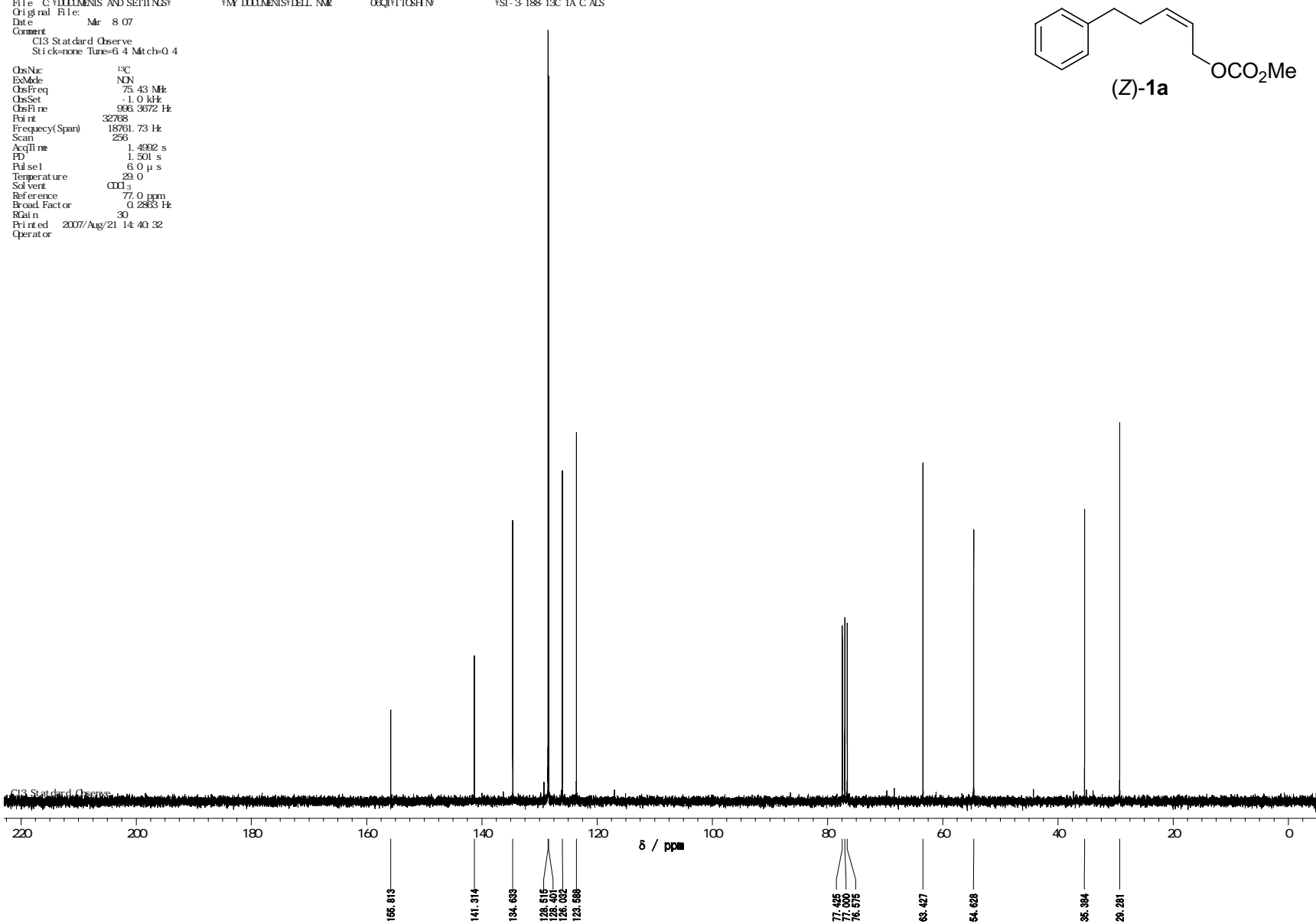

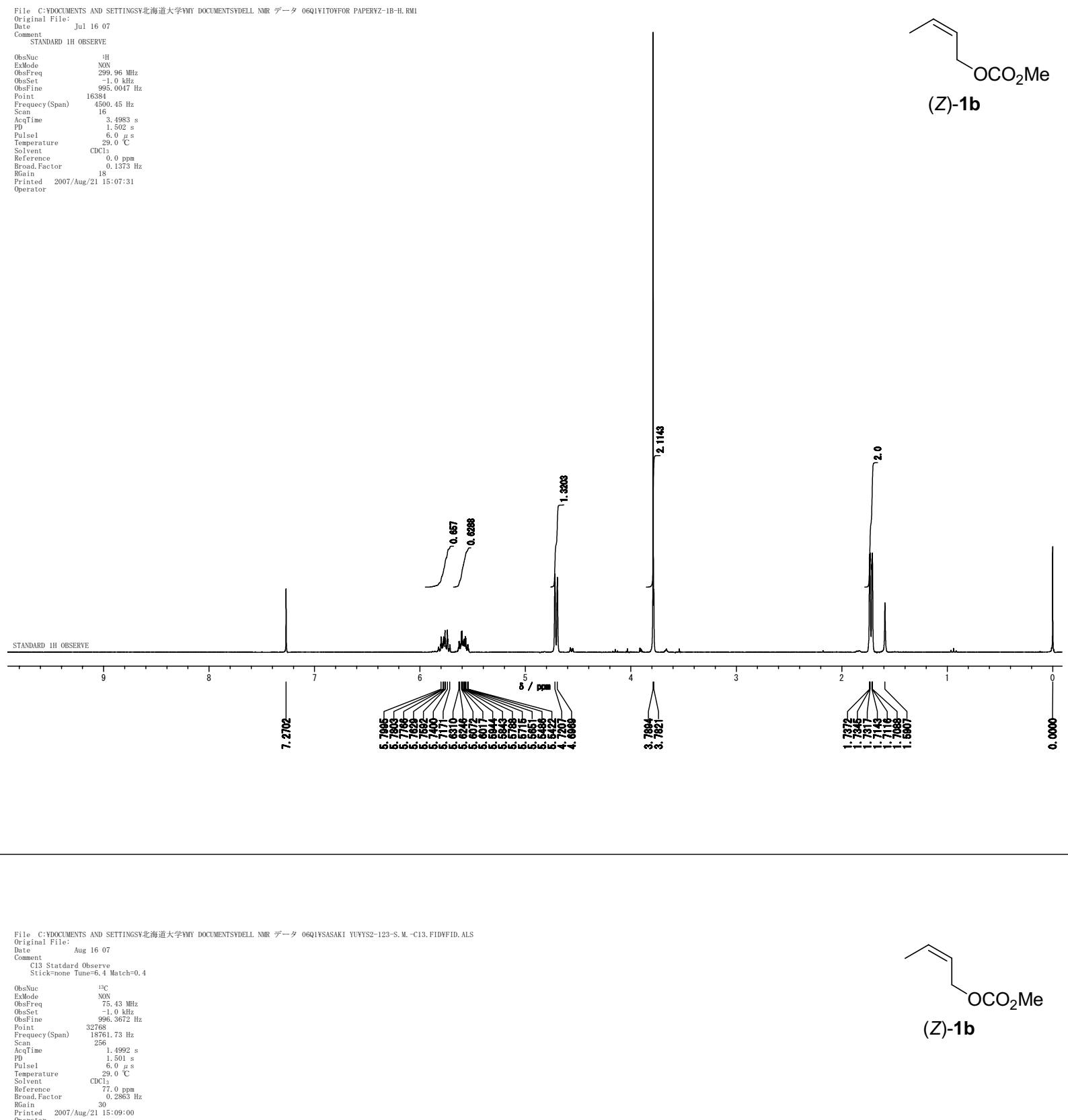

(Z)-1b

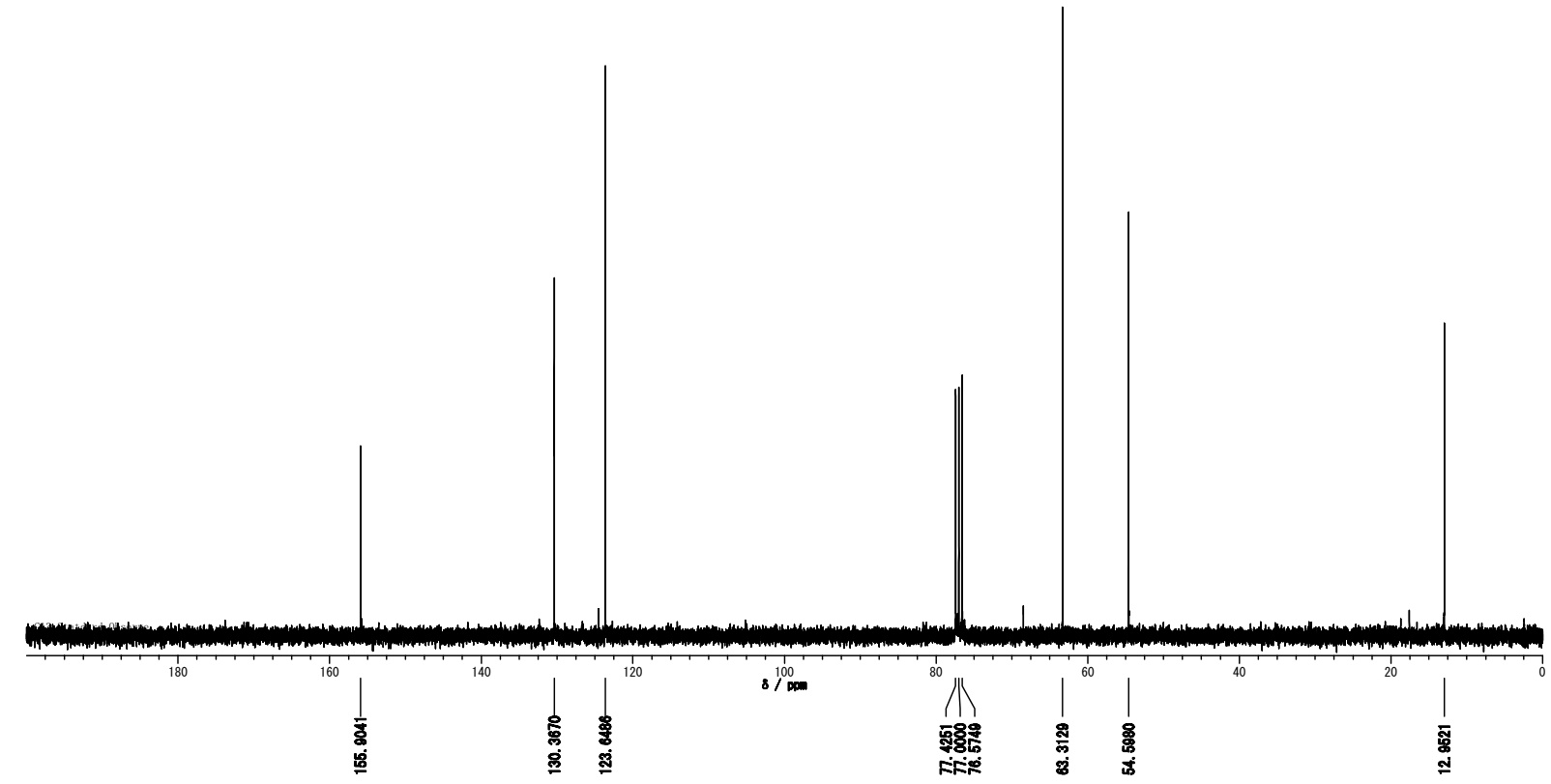



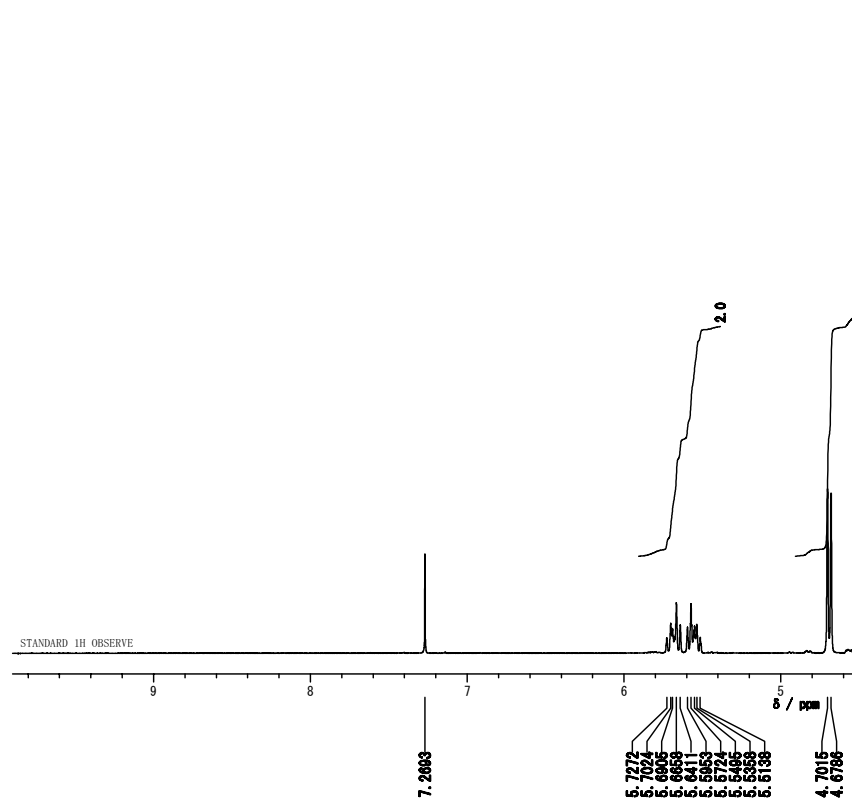

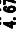

象的
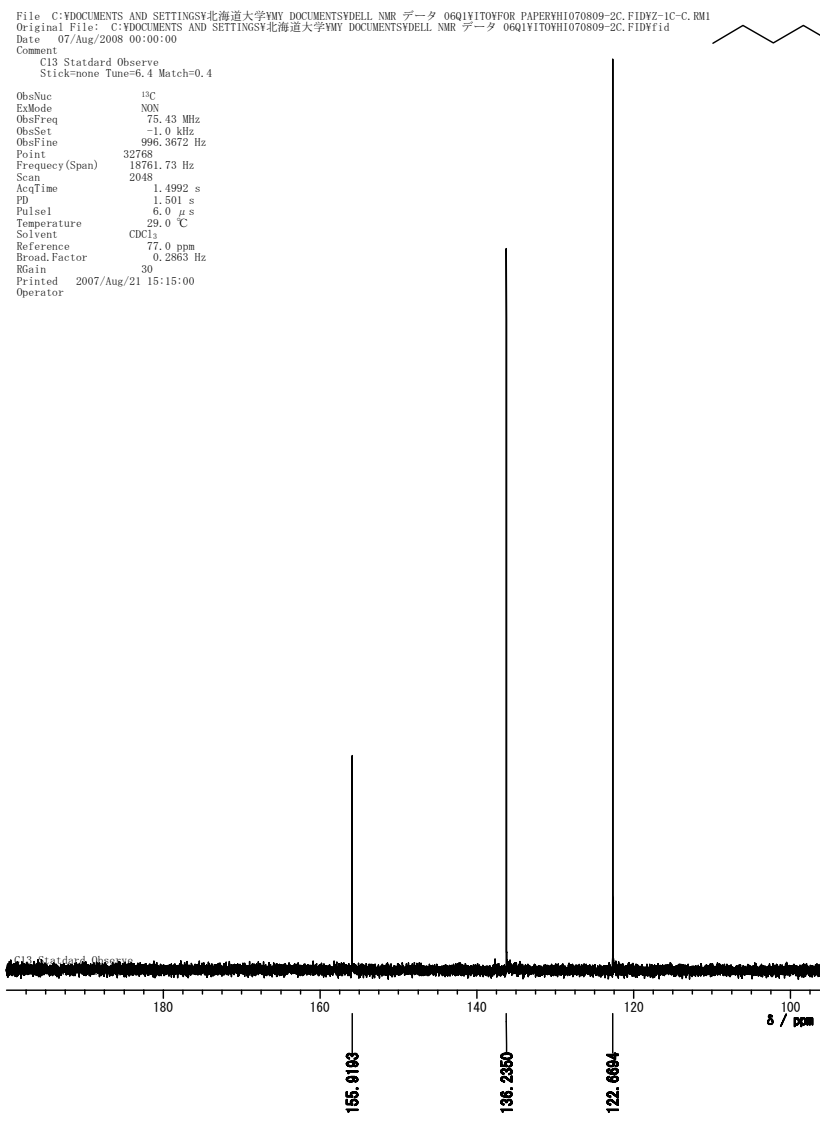

${ }^{80}$

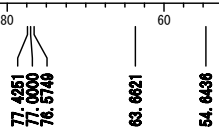

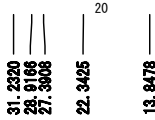




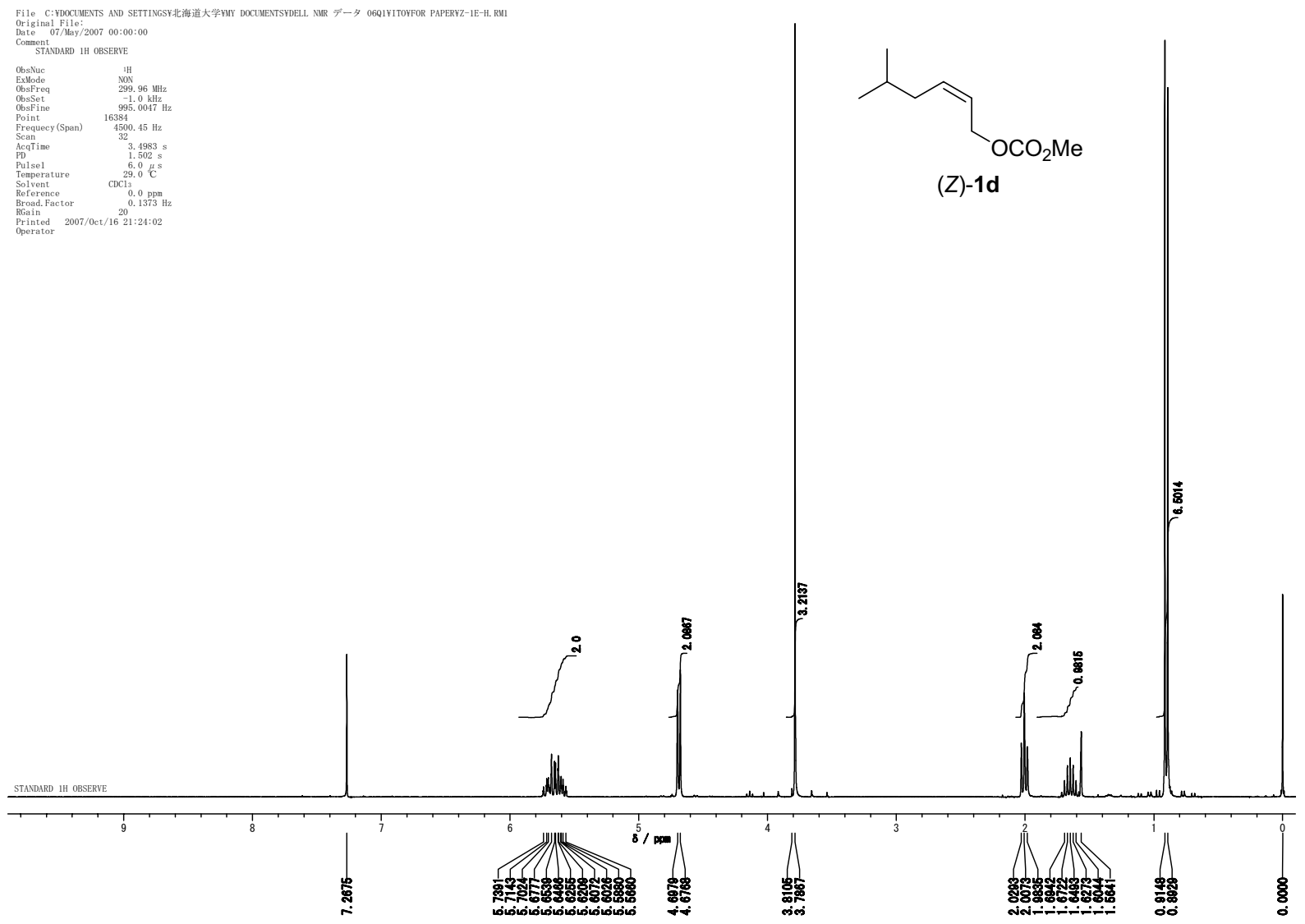

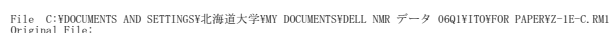

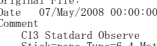
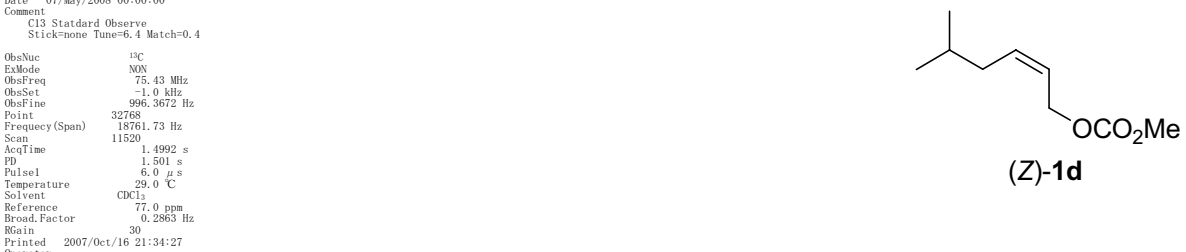

(Z)-1d

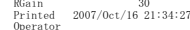

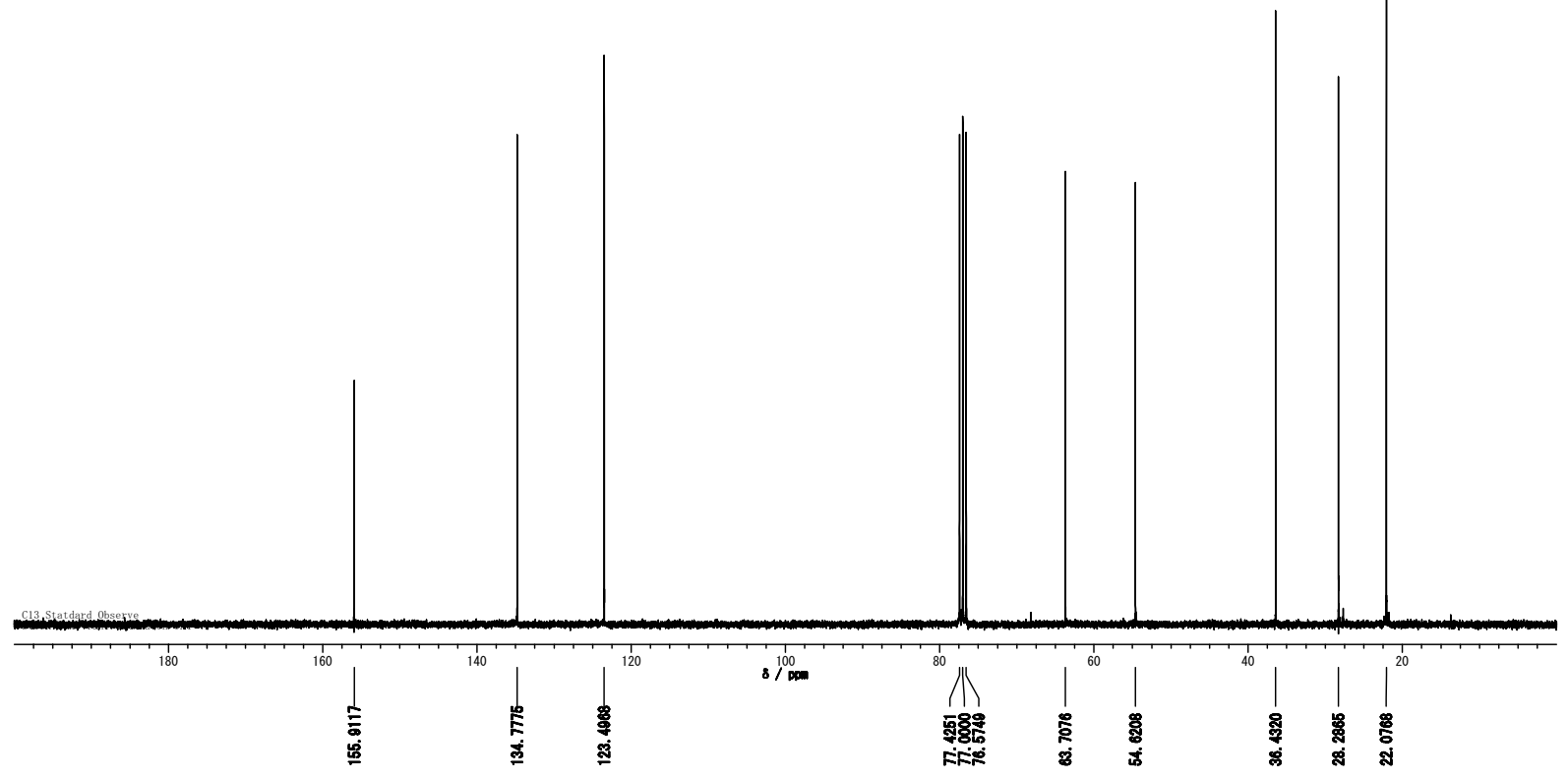




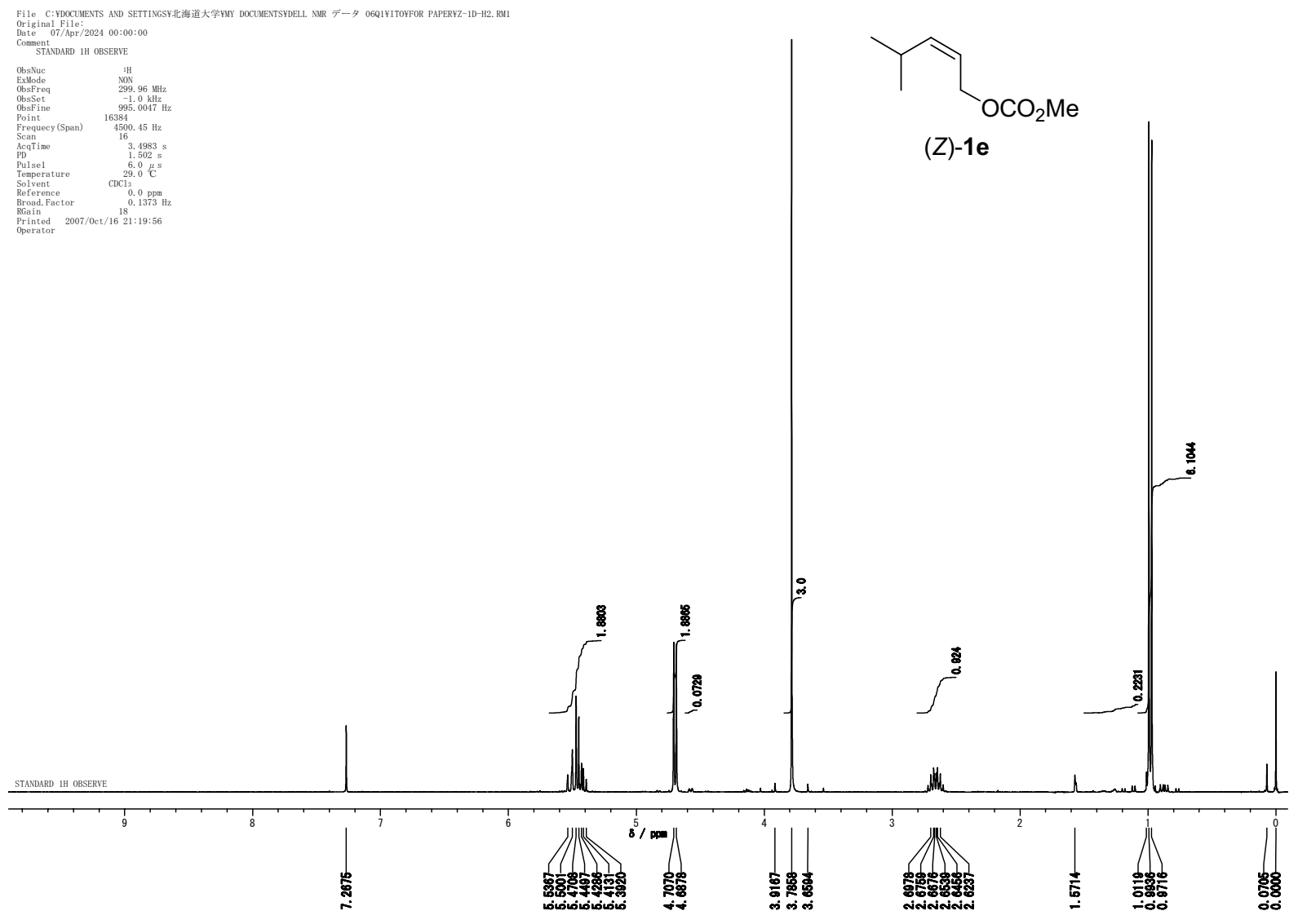

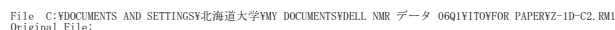
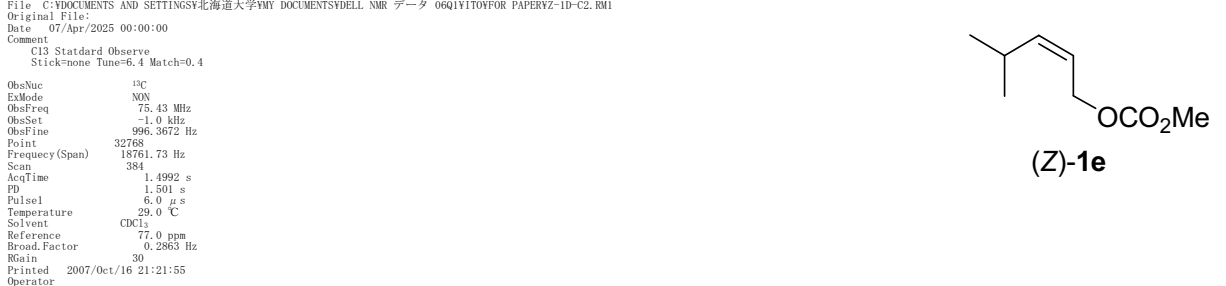

(Z)-1e

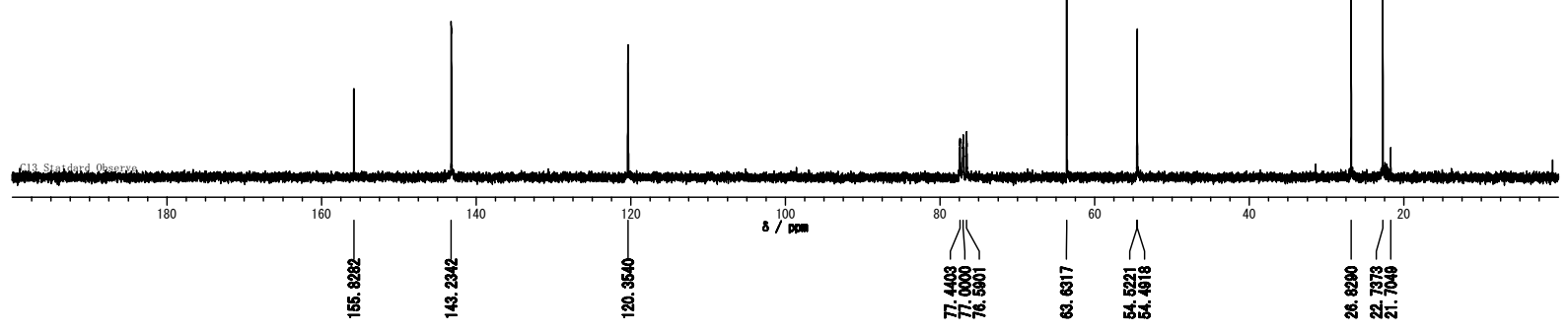




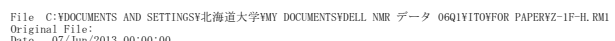
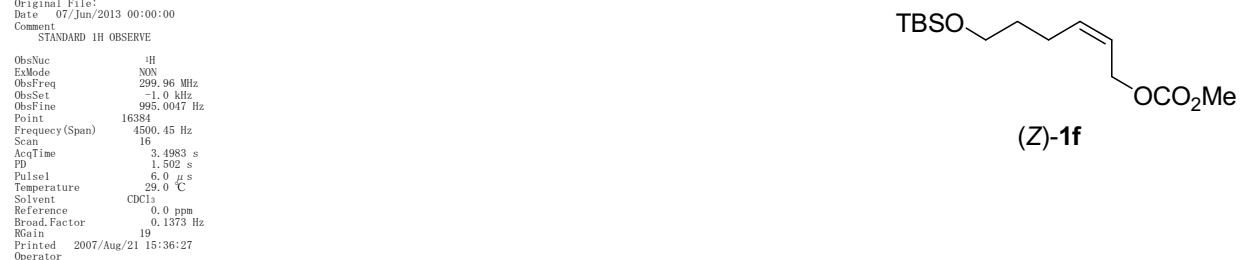

(Z)-1f
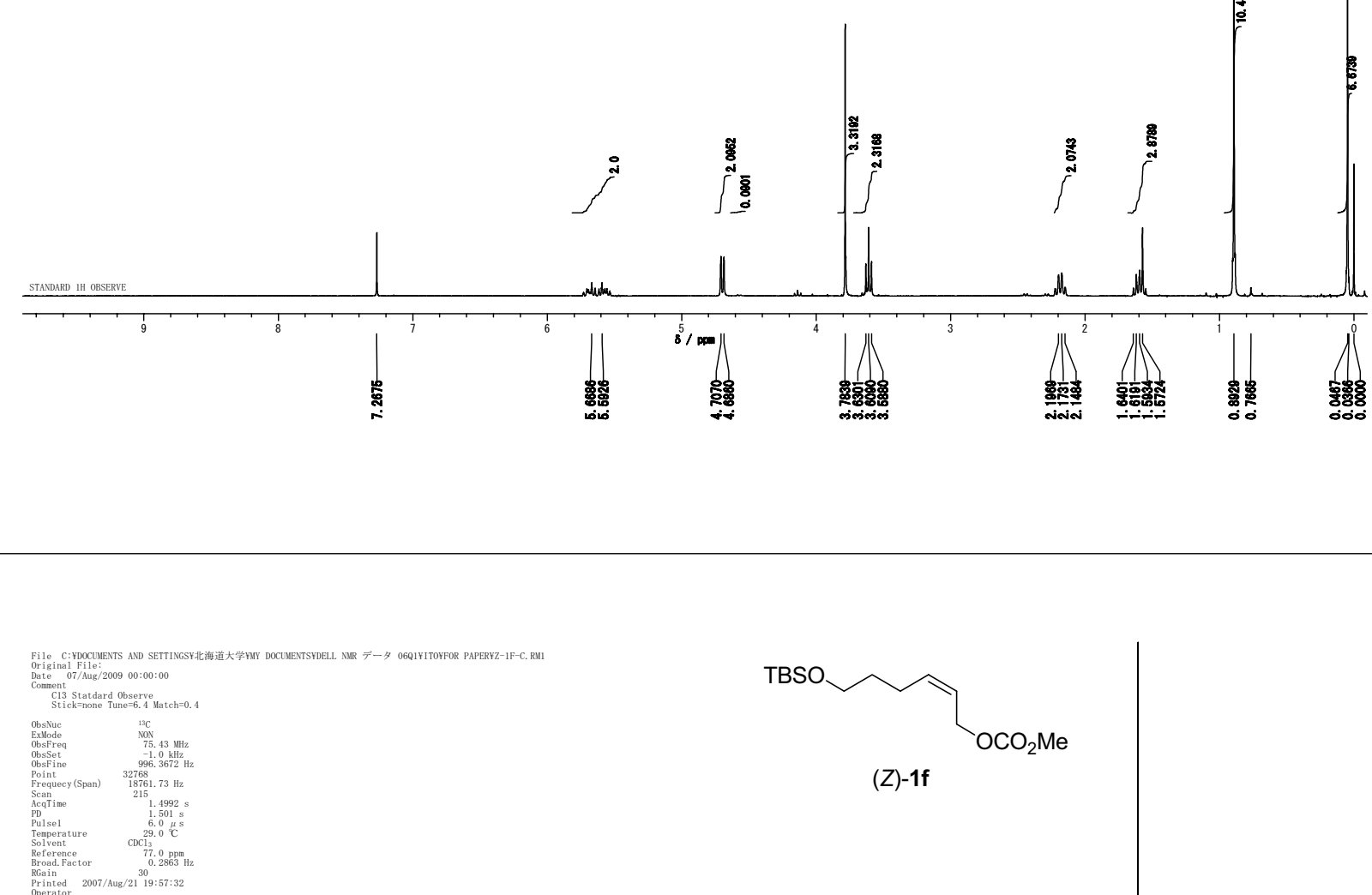

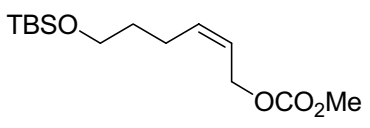

(Z)-1f

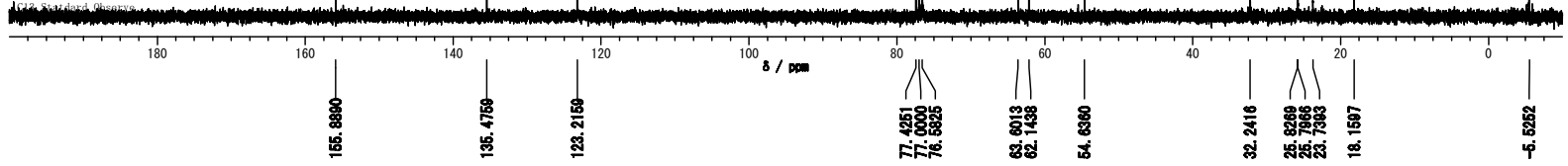



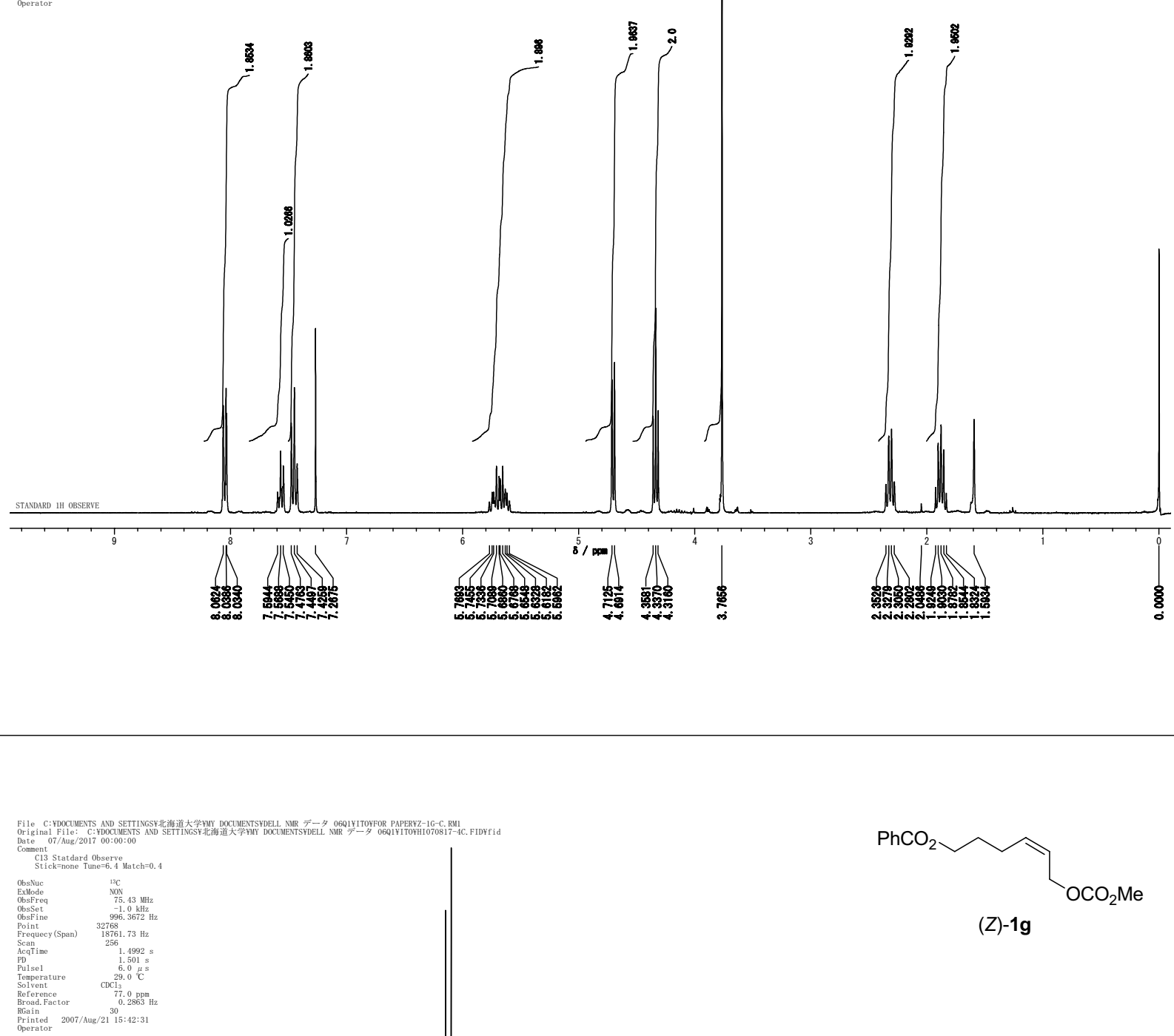

(Z)-1g

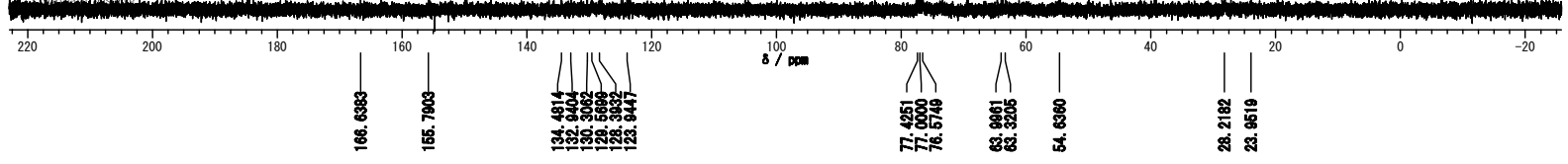



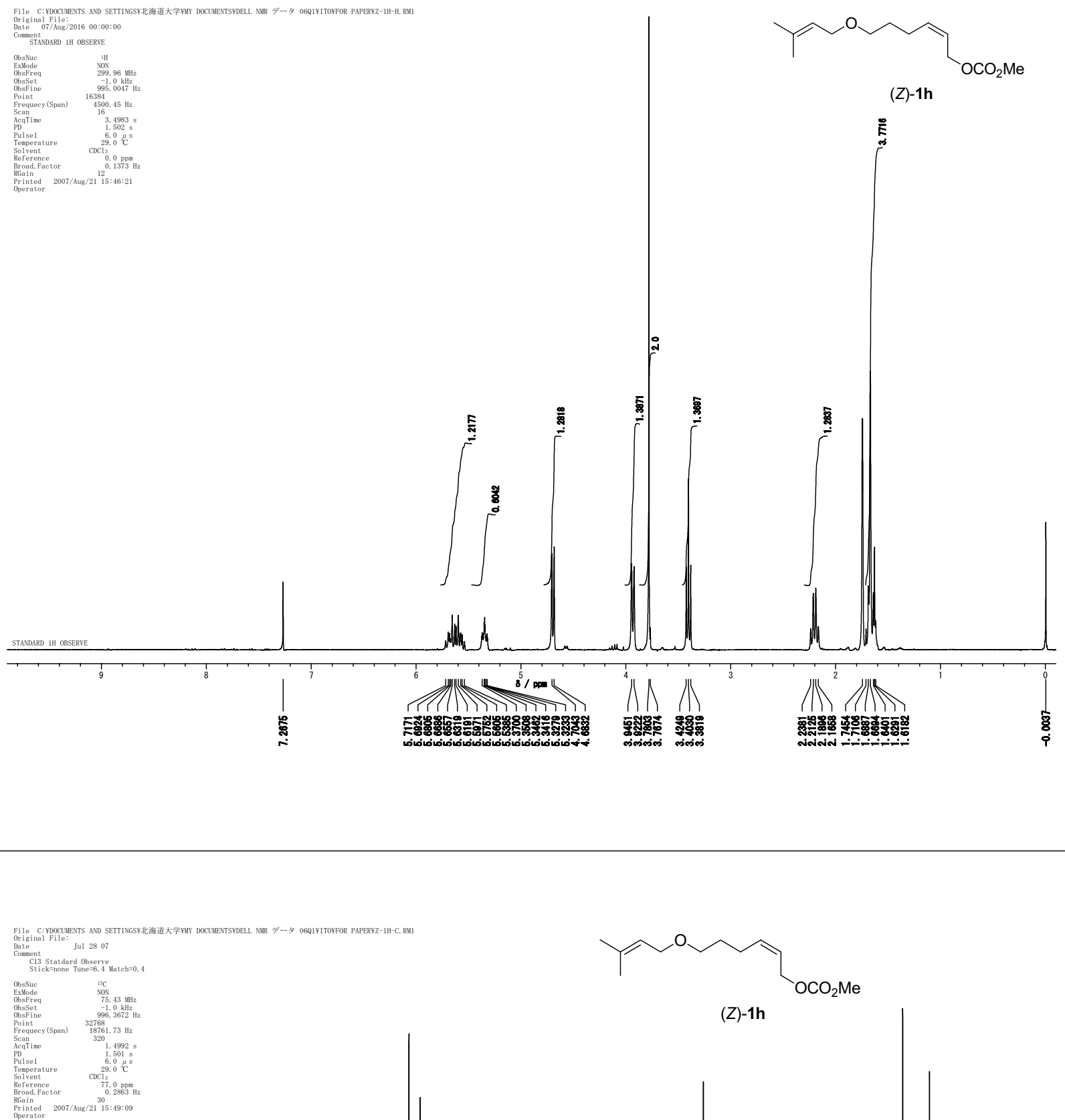

(Z)-1h

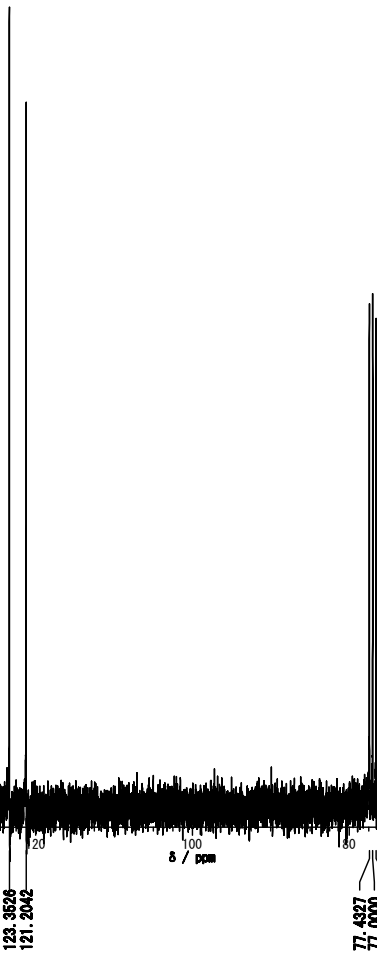


<smiles>C=CC(Br)CCc1ccccc1</smiles>

(S)-3a
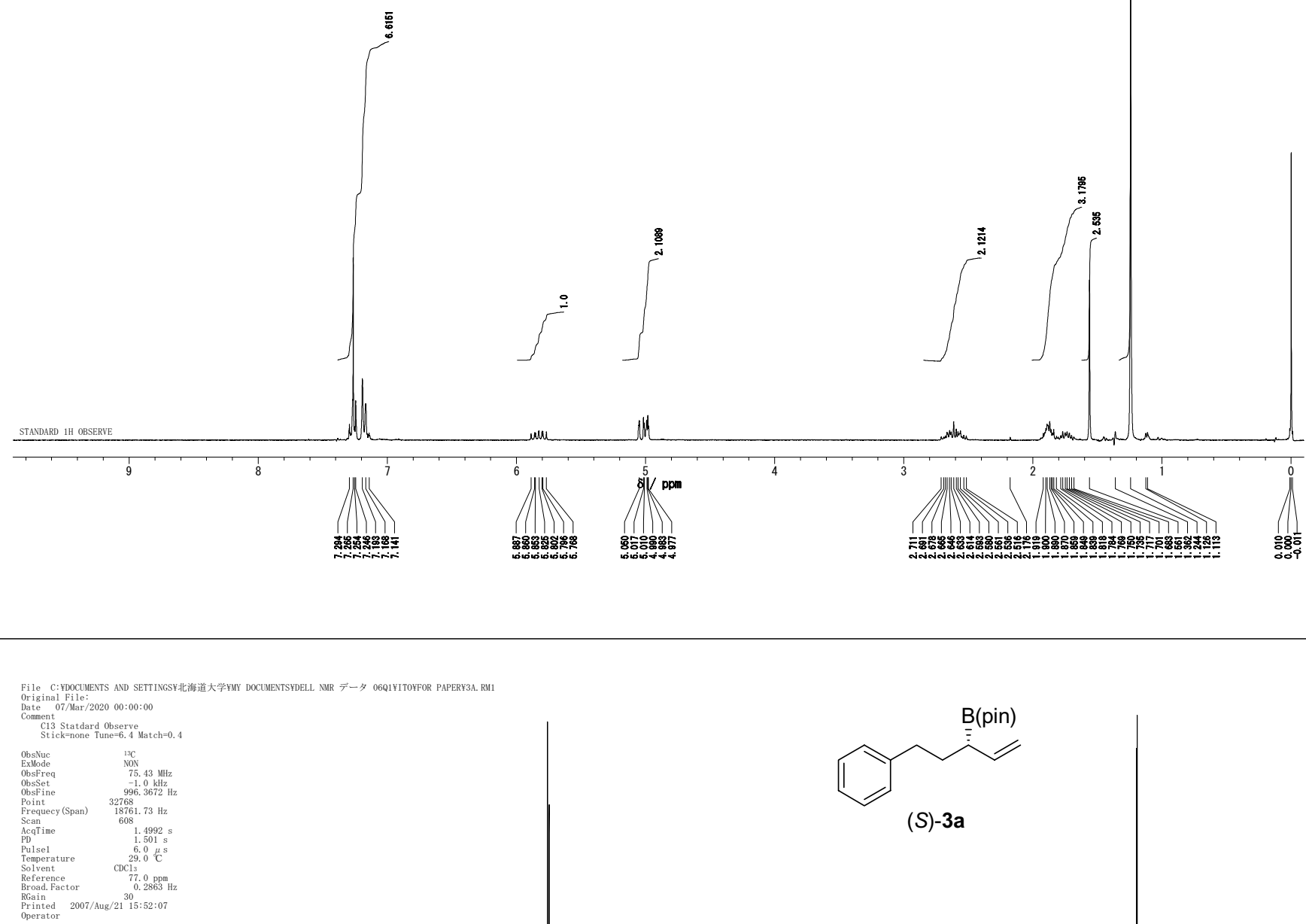

(S)-3a

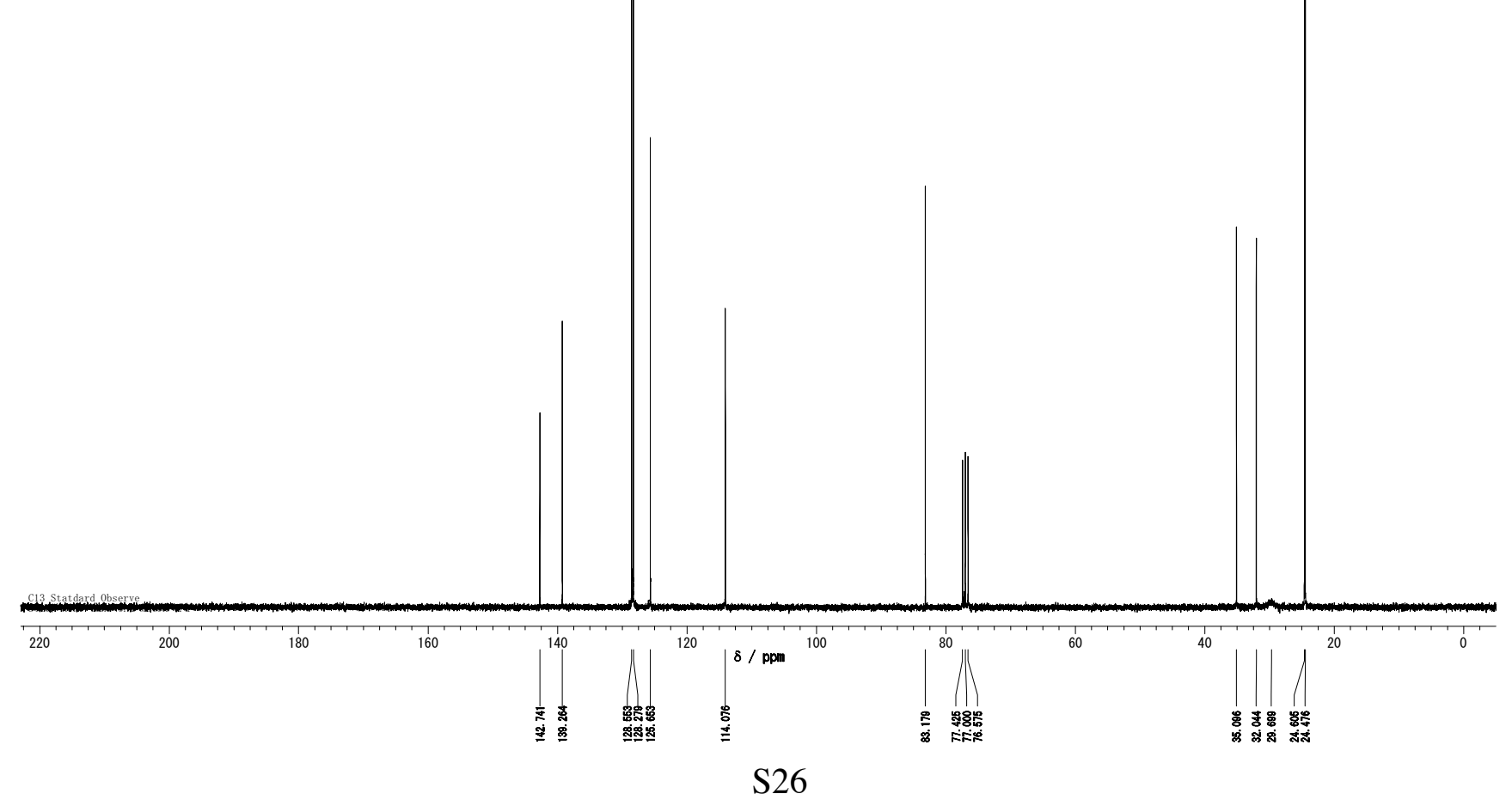




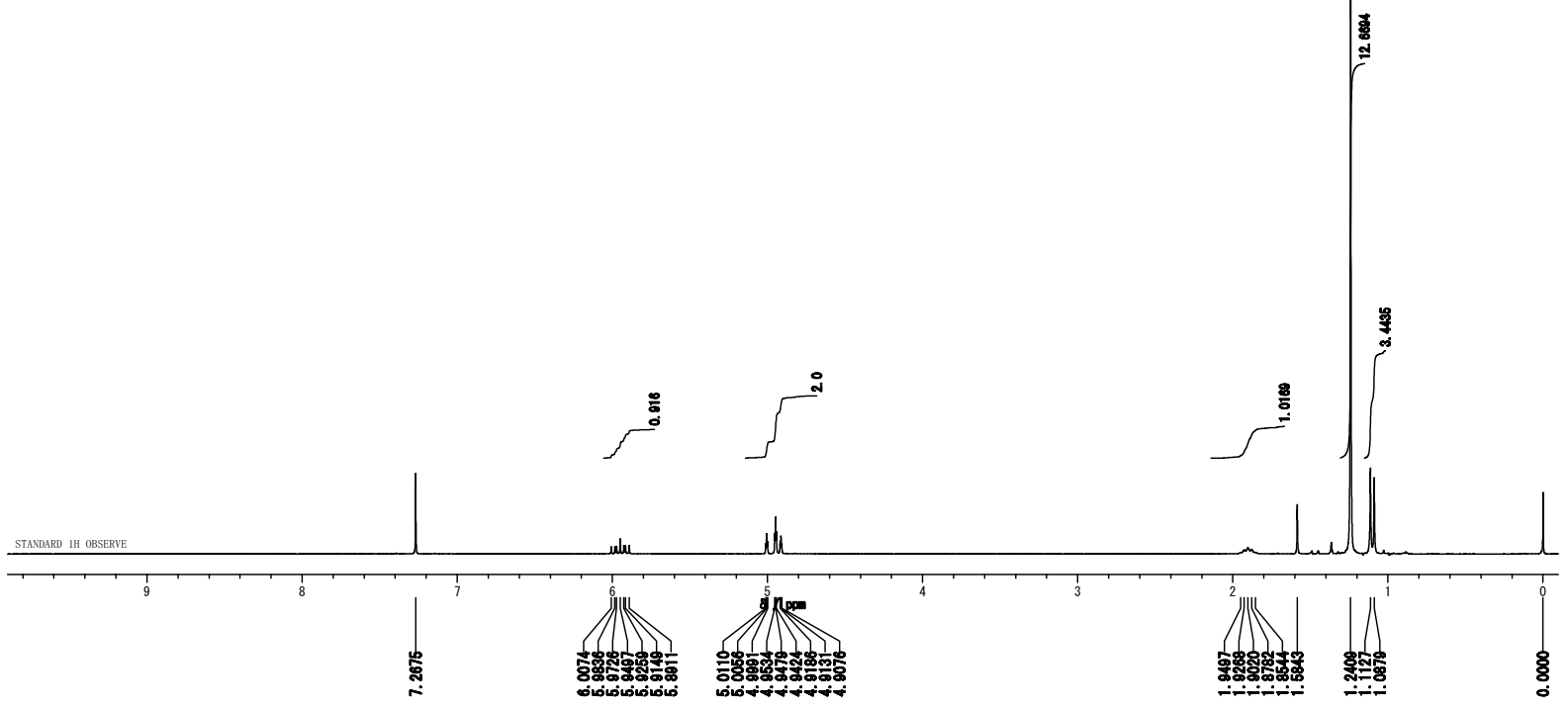

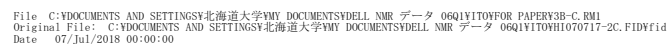

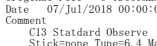

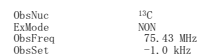

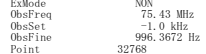

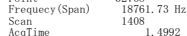

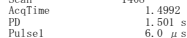

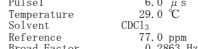

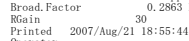

(S)-3b

(2)

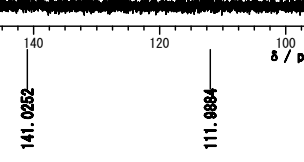

100

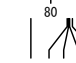

곡ำ

SEFiFi

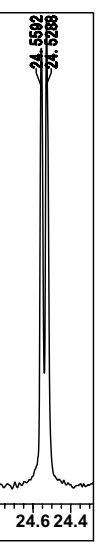




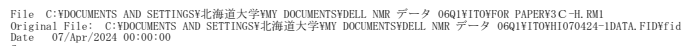

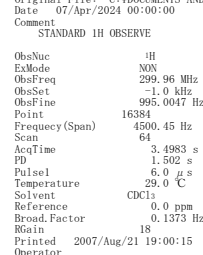

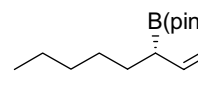

(S)-3c
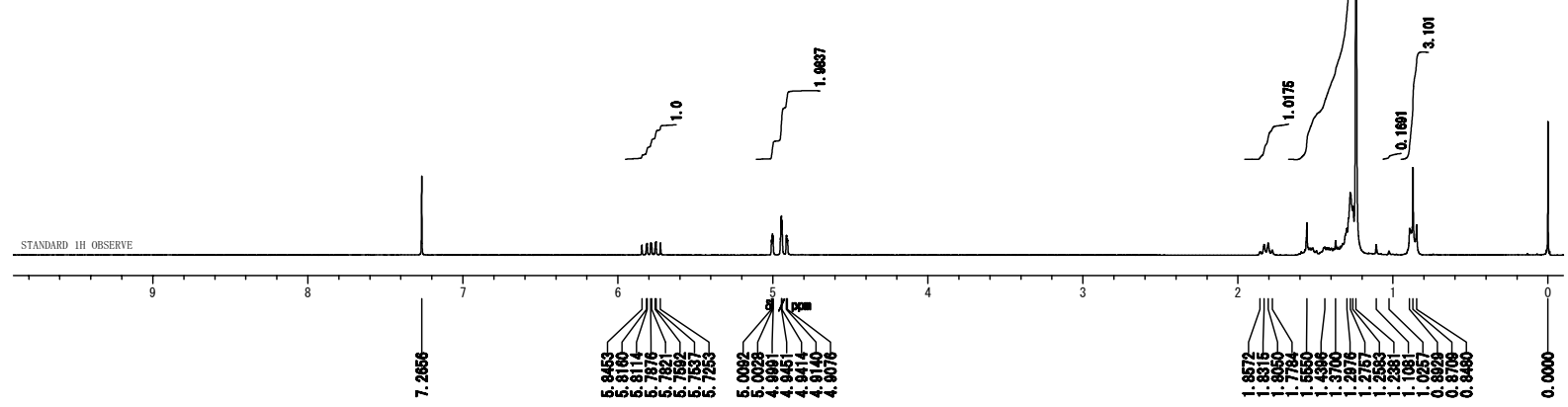

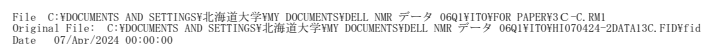

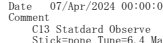

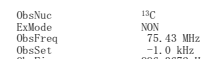

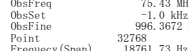

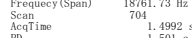

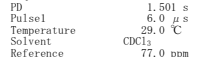

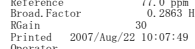

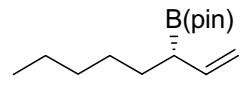

(S)-3c

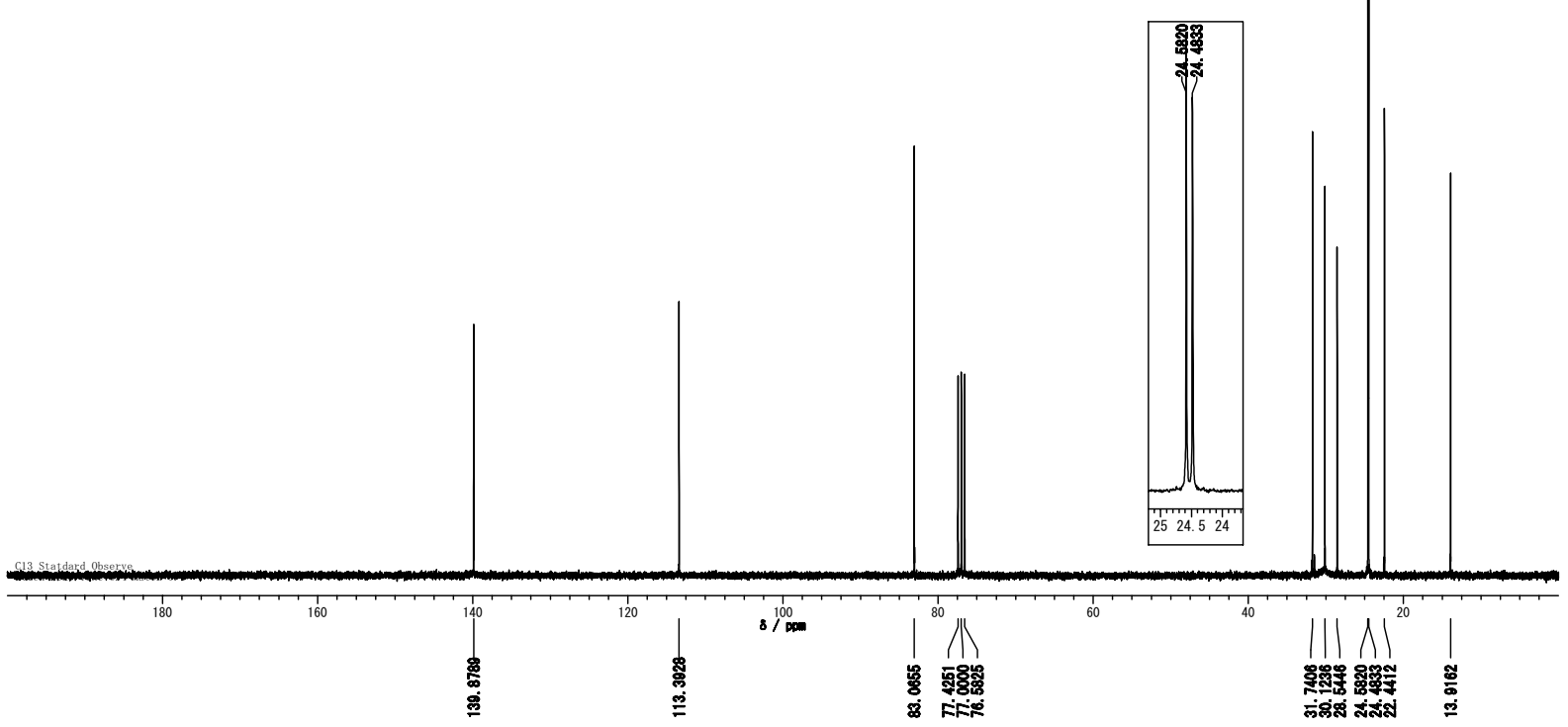



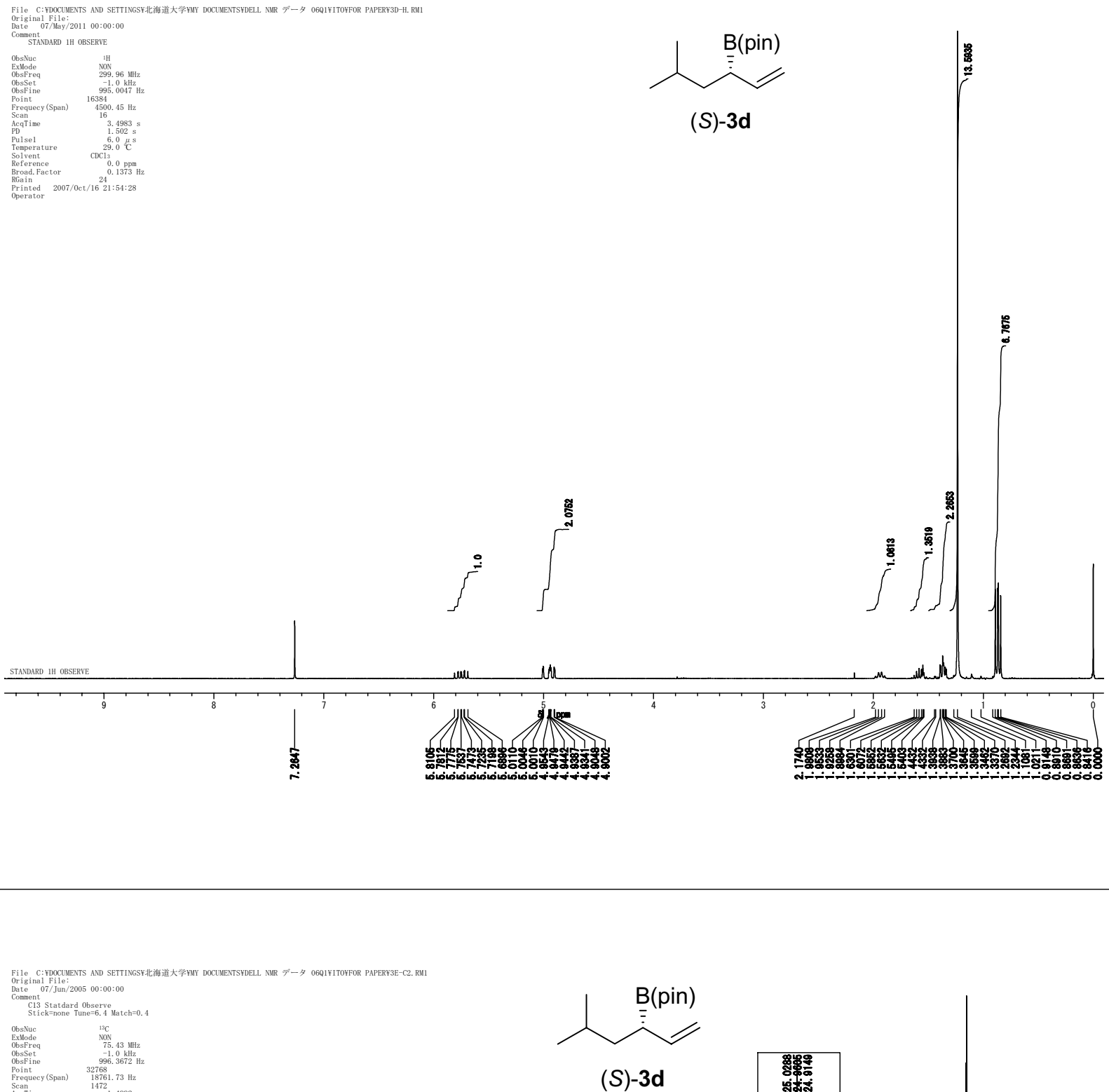

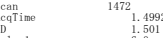

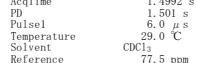

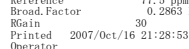

(S)-3d
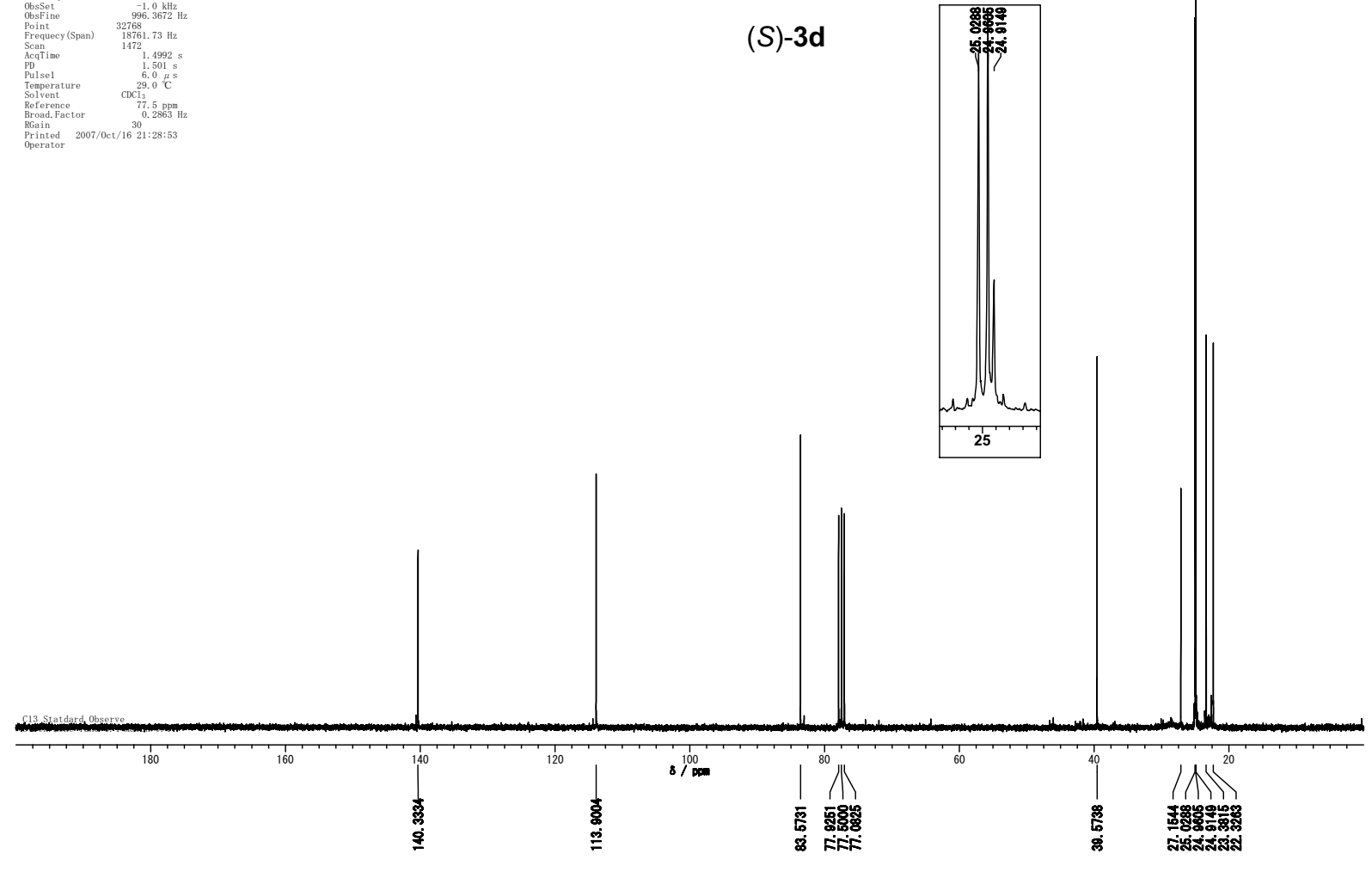

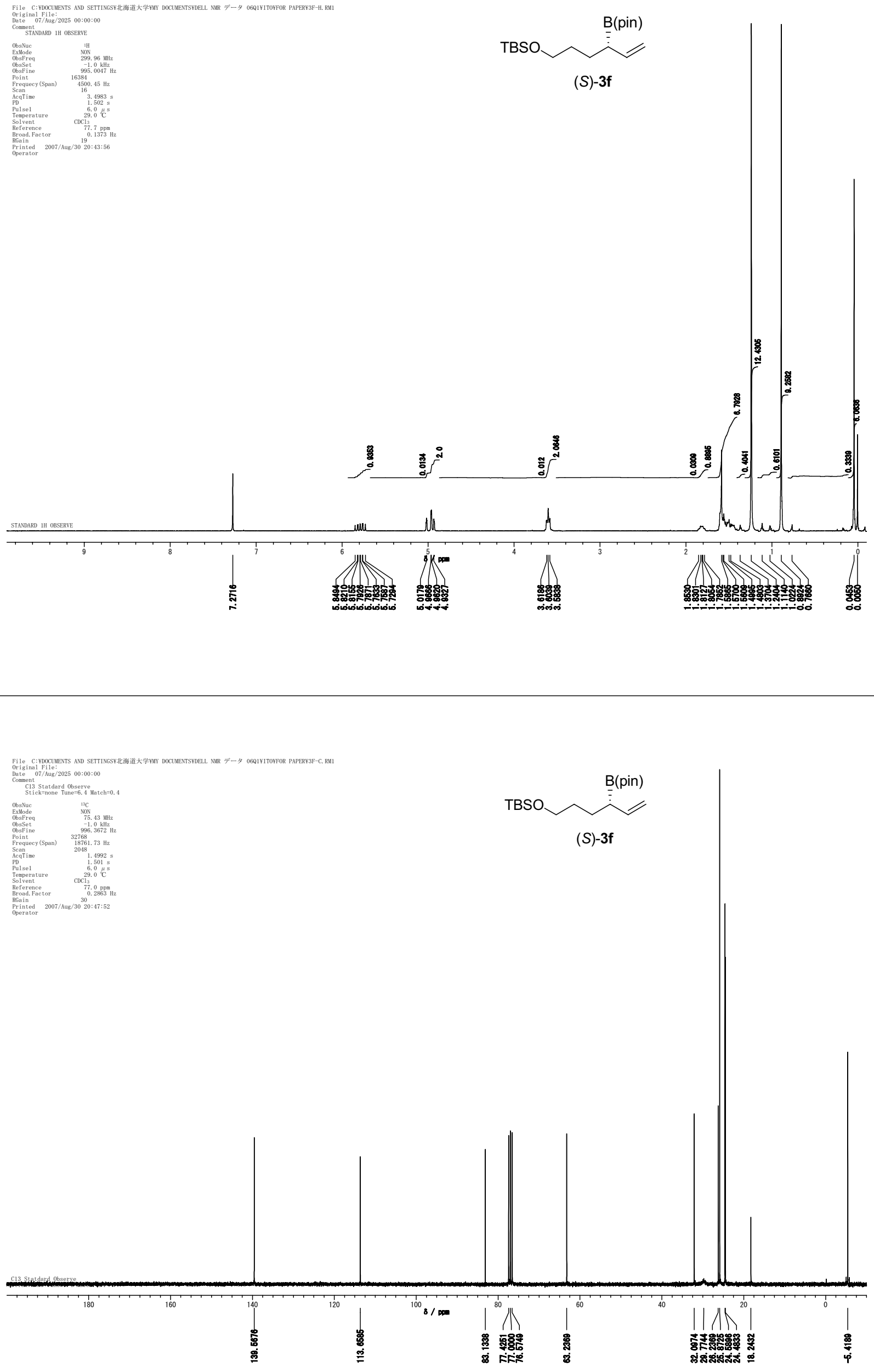
$\mathrm{PhCO}_{2} \overbrace{}^{\mathrm{B}(\text { pin })}$

(S)-3g
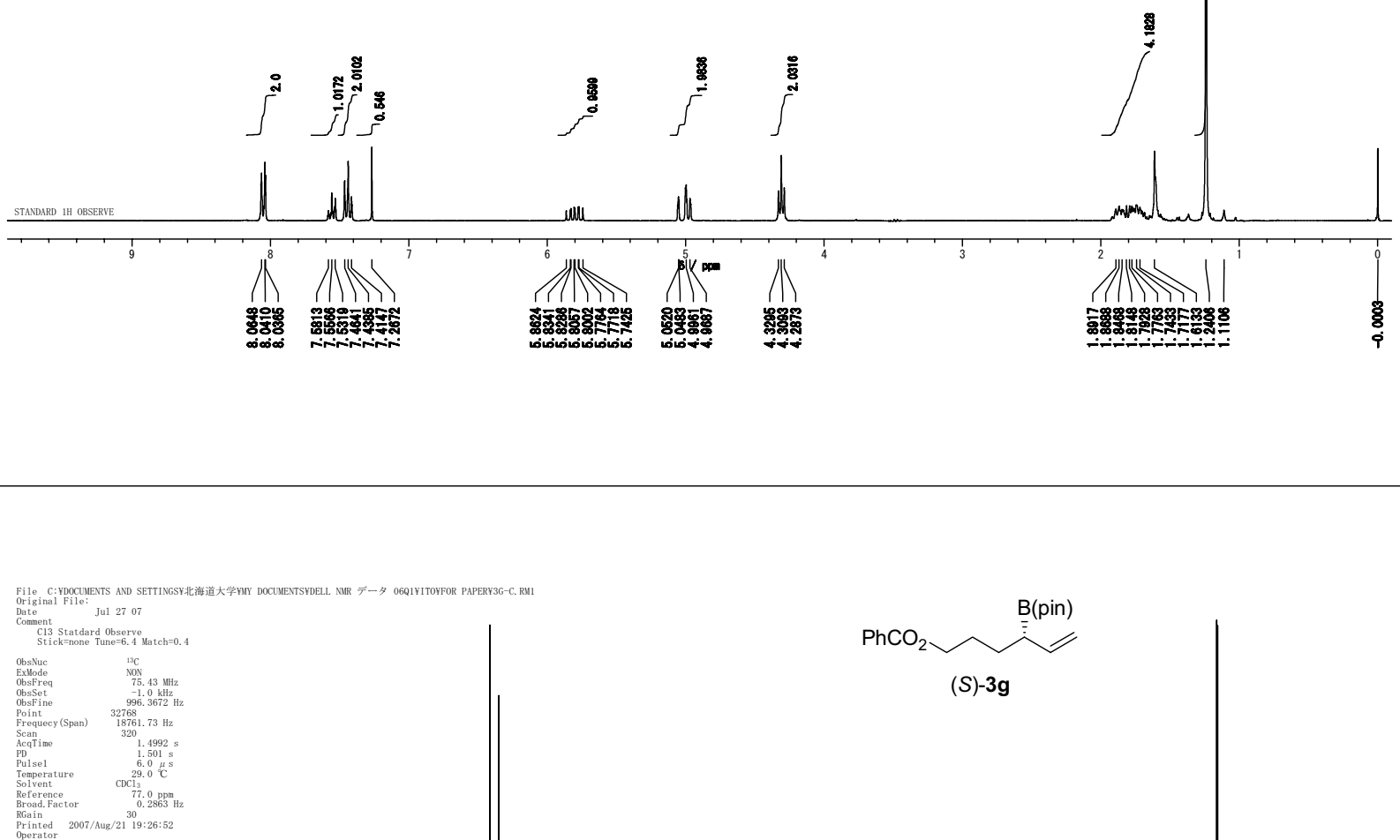

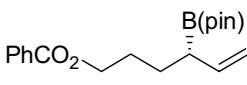

(S)-3g

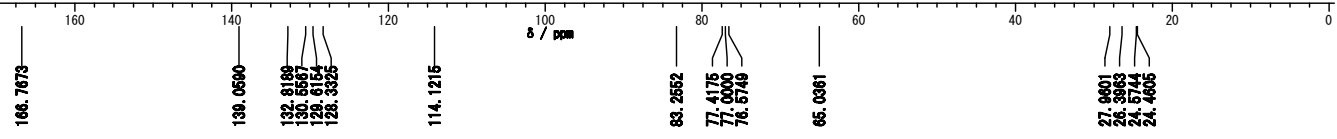



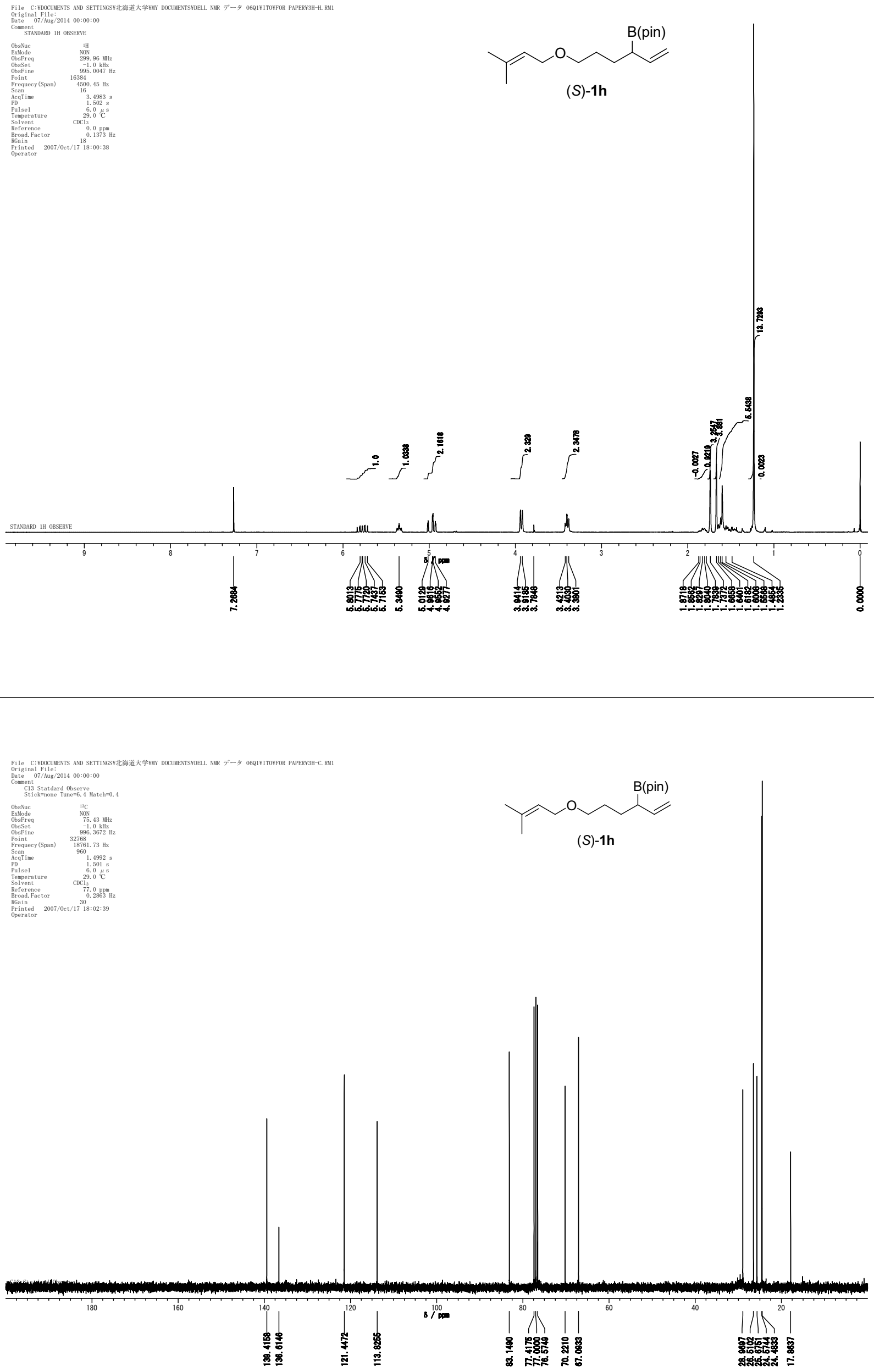

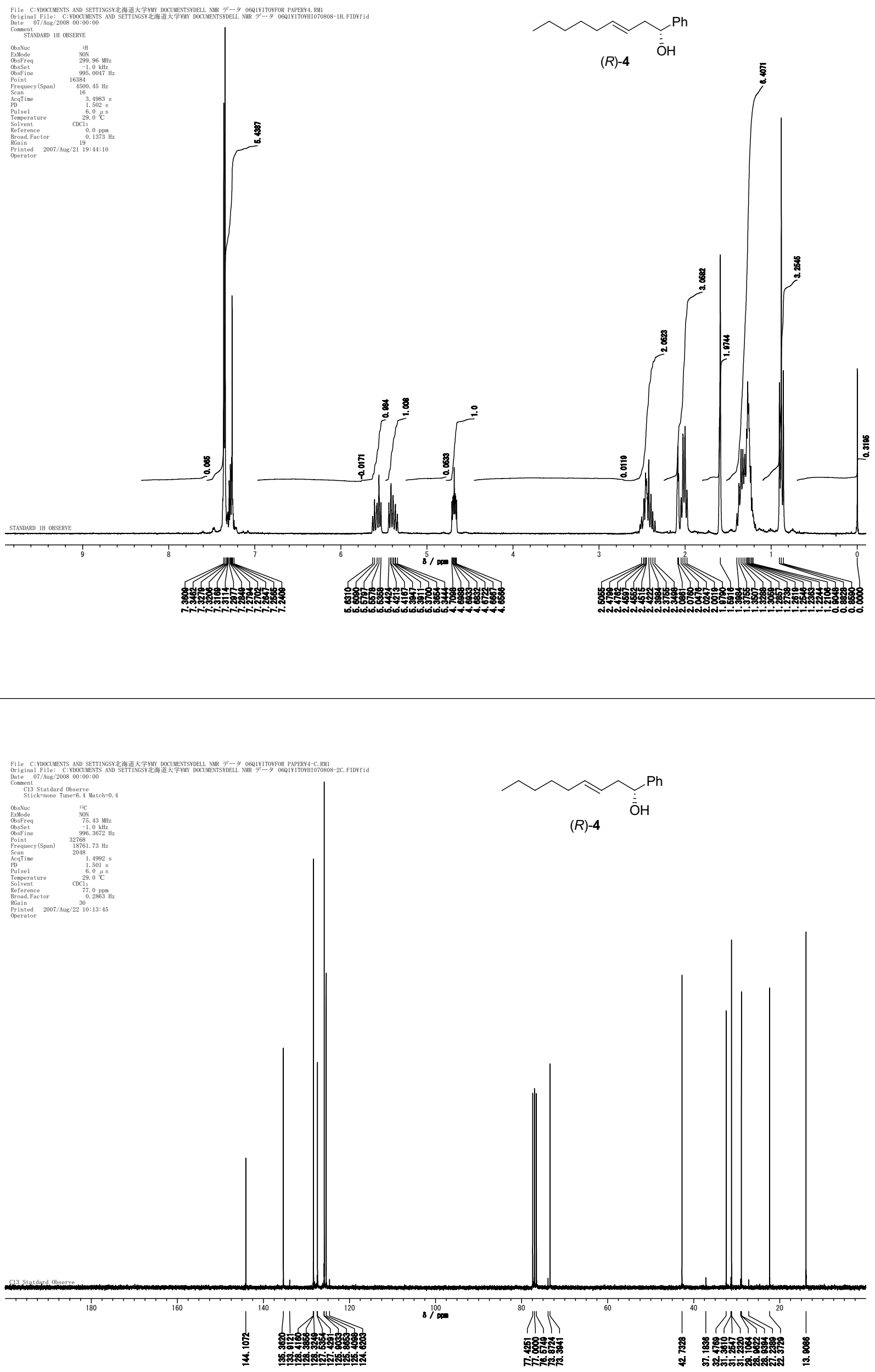